\title{
Palinotaxonomia da seção cretácea a neogena da Bacia de Pelotas, Brasil: cistos de dinoflagelados da Ordem Peridiniales
}

\author{
Eduardo PREMAOR ${ }^{1}$, Paulo A. SOUZA ${ }^{1}$, Elizabete P. FERREIRA ${ }^{2}$, G. Raquel GUERSTEIN ${ }^{3}$ \& \\ Mitsuru ARAI ${ }^{4}$
}

1 Laboratório de Palinologia Marleni Marques Toigo, Programa de Pós-graduação em Geociências, Instituto de Geociências, Universidade Federal do Rio Grande do Sul. Av. Bento Gonçalves, 9.500, CEP 91.540-000, Porto Alegre, RS, Brasil (eduardopremaor@gmail.com, paulo.alves.souza@ufrgs.br).

2 Centro de Pesquisas e Desenvolvimento Leopoldo A. M. Mello, Petrobras. Av. Horácio Macedo, 950, CEP 21.941-915, Rio de Janeiro, RJ, Brasil (elizabete@petrobras.com.br).

3 Instituto Geológico del Sur, CONICET, Departamento de Geología, Universidad Nacional del Sur. Av. San Juan, 670, CP 8.000, Bahía Blanca, BA, Argentina (raquel.guerstein@uns.edu.ar).

4 Centro de Geociências Aplicadas ao Petróleo, Instituto de Geociências e Ciências Exatas, Universidade Estadual Paulista Júlio de Mesquita Filho. Av. 24 A, 1515, CEP 13.506-900, Rio Claro, SP, Brasil (mitsuru.arai@gmail.com).

Recebido em 01/2017. Aceito para publicação em 11/2017.

Versão online publicada em 16/3/2018 (www.pesquisasemgeociencias.ufrgs.br)

\begin{abstract}
Resumo - Grande parte do conhecimento micropaleontológico da Bacia de Pelotas é referente a microfósseis de parede calcária, o que justifica a necessidade de levantamento sistemático de dados palinológicos. Este trabalho apresenta os resultados palinológicos obtidos a partir da análise de 535 amostras de dois poços perfurados na porção offshore da Bacia de Pelotas, incluindo depósitos do Cretáceo ao Neogeno. Os conjuntos palinológicos reconhecidos são ricos e diversificados, constituídos por esporos (briófitas e pteridófitas), grãos de pólen (gimnospermas e angiospermas), cistos de dinoflagelados, palinoforaminíferos, escolecodontes e esporos de fungos. Como consequência do grande volume de dados gerados, se fez necessário, para efeito de publicação, a compartimentação das informações. Nesta primeira contribuição, cistos de dinoflagelados da Ordem Peridiniales são apresentados, com descrições e fotomicrografias. Dentre os 220 táxons de cistos de dinoflagelados identificados, 76 são referentes à Ordem Peridiniales, compreendendo 31 gêneros, 72 espécies, uma subespécie, além de dois táxons sem determinação específica. Ressaltam-se ainda 24 espécies documentadas pela primeira vez para o Brasil. Considerações bioestratigráficas e paleobiogeográficas são discutidas sinteticamente, tendo como base as associações reconhecidas para o Cretáceo Superior e o Neogeno na margem atlântica americana e regiões próximas ao Círculo Polar Antártico.
\end{abstract}

Palavras-chave: palinologia, peridinioides, taxonomia, margem continental brasileira, Cretáceo Superior, Neogeno.

\begin{abstract}
Palynotaxonomy of the Cretaceous to Neogene section of the Pelotas Basin, BRAZIL: DINOFLAGELLATE CYSTS OF PERIDINIALES ORDER . Major part of micropaleontological knowledge of the Pelotas Basin is related to calcareous microfossils, justifying a systematic survey on the palynological data. This paper presents the taxonomic results from the study of 535 stratigraphic samples of two wells drilled in the offshore portion of the Pelotas Basin, enclosing Cretaceous to Neogene deposits. Palynological assemblages analized are diverse and abundant, consisting of spores (mosses and ferns), pollen grains (gymnosperms and angiosperms), dinoflagellate cysts, palynoforaminifers, scolecodonts and fungal spores. As a result of the large volume of generated data, it was necessary, for effect of publication, the compartimentation of the information. In this first part, dinoflagellate cysts Peridiniales Order are presented, with descriptions and photomicrographs. Among the 220 taxa identified of dinoflagellate cyst, 76 belong to the Peridiniales Order, attributable to 31 genera, 72 species, a subspecie, beyond two taxa described without specific determination. A considerable number of 24 species reported for the first time for Brazilian basins is highlighted. Biostratigraphic and paleobiogeographic considerations are synthetically discussed, based on the associations recognized for Late Cretaceous and Neogene in American Atlantic margin and regions close to the Antartic Polar Circle.
\end{abstract}

Keywords: palynology, peridinians, taxonomy, Brazilian continental margin, Upper Cretaceous, Neogene. 


\section{Introdução}

Os dinoflagelados (Divisão Dinoflagellata, Classe Dinophyceae) são organismos unicelulares comumente representados no registro paleontológico sob a forma de cistos de parede orgânica pertencentes às ordens Gonyaulacales e Peridiniales (Fensome et al., 1993). Tais ordens apresentam maior diversidade e abundância particularmente nos depósitos siliciclásticos plataformais do Cretáceo ao Neogeno (Fensome et al., 1996), e são encontrados geralmente associados a outros microfósseis de parede calcária e silicosa, tais como foraminíferos planctônicos e cocolitoforídeos. Esses microfósseis são utilizados para fins de interpretação paleoambiental e para o estabelecimento de zoneamentos bioestratigráficos de alta resolução em fácies marinhas, permitindo atribuições de idades, correlações inter-regionais e globais entre distintas províncias paleobiogeográficas (Stover et al., 1996; Jones, 2011; Bijl et al., 2013).

No Brasil, os dados sobre cistos de dinoflagelados são advindos de depósitos relacionados às bacias da Margem Continental Atlântica, com idades a partir do final do Eocretáceo (Regali et al., 1974a, 1974b; Arai et al., 2000; Lana \& Pedrão, 2000; Arai, 2005, 2014). Contudo, grande parte do conhecimento taxonômico e bioestratigráfico destes microfósseis está restrita a relatórios internos de companhias de exploração de hidrocarbonetos e trabalhos inéditos (e.g., Botelho Neto, 1996; Lana, 1997; Sarkis, 2002; Ferreira, 2004; Arai, 2007).

Para a Bacia de Pelotas, mais meridional do território brasileiro, são conhecidas somente quatro ocorrências de palinomorfos publicadas, envolvendo seções de subsuperfície, de intervalos estratigráficos relativamente estreitos, posicionados no Campaniano (Premaor et al., 2010), Eoceno (Fischer et al., 2013) e Mioceno/Plioceno (Silva et al., 2011, 2015). Nestas contribuições, cistos de dinoflagelados fósseis selecionados são ilustrados, juntamente com outros palinomorfos de origem continental (esporomorfos) e marinha (e.g., palinoforaminíferos). Desta forma, é demonstrada notável carência de informação palinológicas, o que é válido para a maioria das demais bacias marginais brasileiras, com ausência de descrições e ilustrações, diferentemente do que é conhecido para outras partes do Hemisfério Sul, com destaque para a Austrália (Helby et al., 1987), Antártica (Askin, 1988; Wrenn \& Hart, 1988), Nova Zelândia (Wilson, 1988; Roncaglia et al., 1999; Crouch et al., 2014) e Argentina (Guerstein \& Junciel, 2001; Guler \& Guerstein, 2003; Guerstein et al., 2008).
Este trabalho constitui parte dos resultados taxonômicos obtidos a partir de uma amostragem extensiva realizada na Bacia de Pelotas (Premaor, 2016), em depósitos do Cretáceo (Albiano) ao Neogeno (Plioceno). Como consequência do grande volume de dados gerados, se fez necessário, para efeito de publicação, a compartimentação das informações. Nesta primeira contribuição, cistos de dinoflagelados da Ordem Peridiniales são apresentados, com descrições e fotomicrografias.

\section{2 Área, materiais e métodos}

\subsection{Localização da área e contexto geológico}

A Bacia de Pelotas está localizada no extremo sul da margem continental brasileira, entre os paralelos $28^{\circ} \mathrm{S}$ e $34^{\circ} \mathrm{S}$ (Fig. 1), tendo o limite norte com a Bacia de Santos, pelo Alto de Florianópolis e, o sul, com o Alto de Polônio, na plataforma continental uruguaia. Distribui-se por $210.000 \mathrm{~km}^{2}$, alcançando a área até a lâmina d'água de $2.000 \mathrm{~m}$, incluindo cerca de $40.000 \mathrm{~km}^{2}$ de área emersa. A origem e a evolução geológica da bacia estão relacionadas ao processo de ruptura do Gondwana e abertura do Oceano Atlântico, com preenchimento sedimentar iniciado a partir do Eocretáceo. Na porção mais proximal, as sequências vulcânicas e sedimentares que compõem a bacia assentam-se sobre a crosta continental e rochas basálticas extrudidas da fase rifte, enquanto na porção mais distal a bacia se estende diretamente sobre o assoalho oceânico (Fontana, 1990; Villwock \& Tomazelli, 1995).

Os trabalhos mais importantes de cunho estratigráfico da bacia foram realizados por Gonçalves et al. (1979), Dias et al. (1994) e Fontana (1996), abordando a subdivisão litoestratigráfica, a estratigrafia de sequências e a sismoestratigrafia, respectivamente. Bueno et al. (2007) apresentaram a carta estratigráfica mais recente integrando dados anteriores. Segundo os mesmos, os depósitos mais basais correspondem à Supersequência Rifte (Cretáceo Inferior), que é dividida em dois estágios: Rifte I, composto por basaltos (Formação Imbituba), e Rifte II, constituído por fácies siliciclásticas. A Supersequência Pós-Rifte (Cretáceo Inferior) representa um momento de subsidência térmica da bacia ocorrida durante o Aptiano, composta por uma suíte vulcânica (Formação Curumim). A Supersequência Drifte é constituída por três unidades: a inferior, representada por depósitos albianos de plataforma rasa; intermediária, relativa a depósitos transgressivos entre o Albiano e o Oligoceno, com arenitos interdigitados; e a 
superior, referente a uma cunha clástica regressiva Drifte, entre o final do Eocretáceo e o Quaternário do Neogeno/Quaternário. Um esboço do compor- é apresentado na figura 2.

tamento estratigráfico das supersequências Rifte e

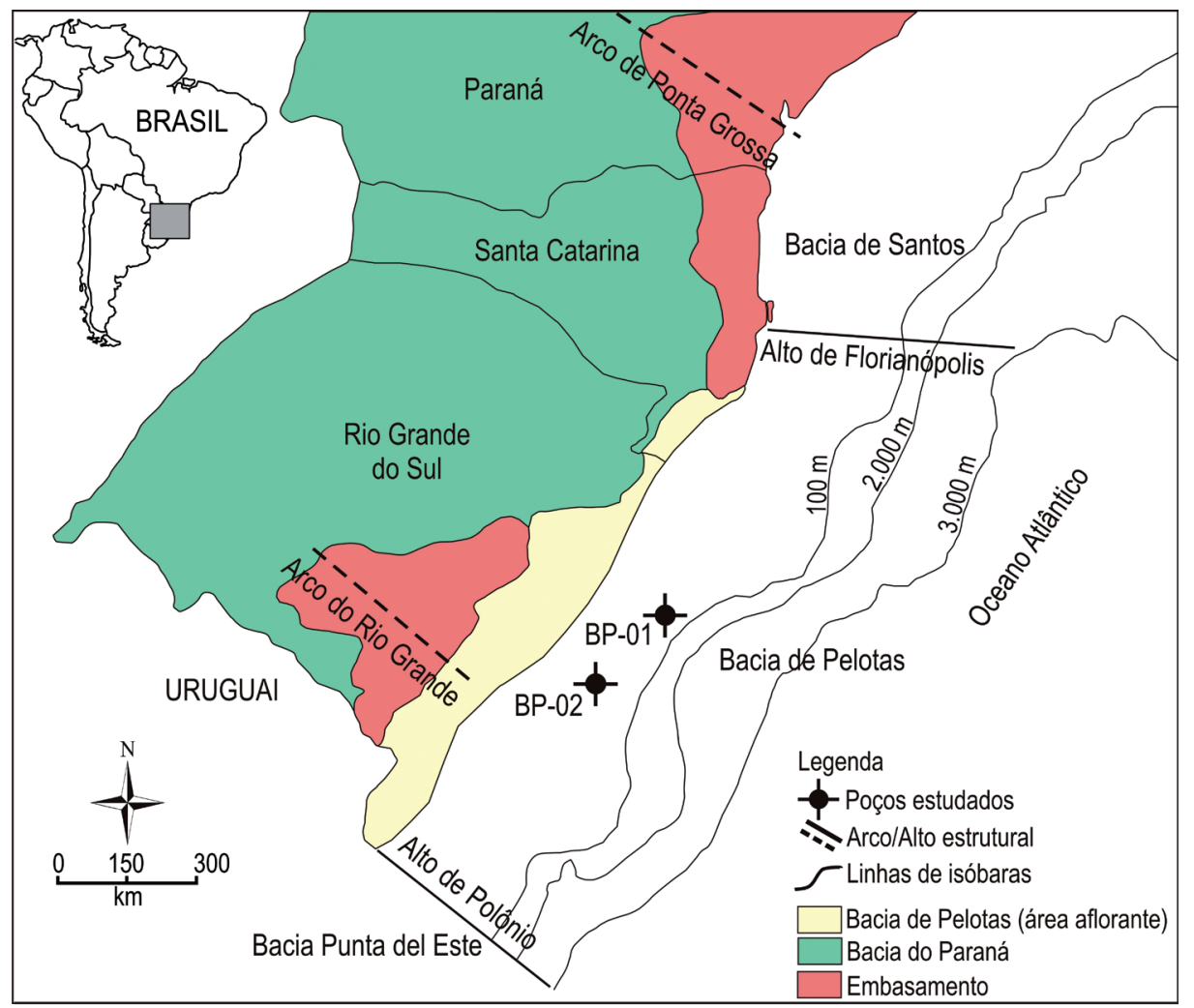

Figura 1. Mapa de localização da área de estudo com indicação das principais feições estruturais e dos poços estudados (modificado de Gomide, 1989 e Milani et al., 2007).

Figure 1. Location map of study area indicating the main structural features and wells studied (modified from Gomide, 1989 and Milani et al., 2007).

\subsection{Contexto paleontológico}

Macrofósseis de vertebrados e icnofósseis foram registrados em várias localidades aflorantes da Planície Costeira do Rio Grande do Sul, porção quaternária da Bacia de Pelotas (Buchmann, 1994). Contudo, a maior parte dos estudos paleontológicos da bacia é relativa a microfósseis (foraminíferos, ostracodes, nanofósseis calcários, diatomáceas e palinomorfos), registrados em seções miocênas e pós-miocênas de poços onshore (e.g., Closs, 1967; Thiesen, 1977; Anjos \& Carreño, 2004; Anjos-Zerfass et al., 2008; Simões et al., 2008; Coimbra et al., 2009; Hermany et al., 2013). Informações paleontológicas de seções pré-miocênas são referentes a foraminíferos (Koutsoukos, 1982), nanofósseis calcários (Gomide, 1989; Guerra et al., 2010, 2012) e ostracodes (Ceolin et al., 2010).

Até recentemente, dados palinológicos sobre a bacia eram de natureza restrita (Daemon, 1969, apud Anjos \& Carreño, 2004), inédita (e.g., Arai, 2007) ou preliminar (e.g., Arai et al., 2006). Somente nos últimos anos foram publicados conjuntos palinológicos das seções pré-miocênas, reconhecidos em poços onshore e offshore da bacia, incluindo elementos de origem continental (esporomorfos) e marinha (cistos de dinoflagelados e palinoforaminíferos), o que representa avanço significativo no conhecimento bioestratigráfico e paleoambiental para seções do Campaniano (Premaor et al., 2010), do Eoceno (Fischer et al., 2013) e Mioceno ao Plioceno (Silva et al., 2011, 2015).

\subsection{Materiais e métodos}

Amostras de calha e de testemunhos de sondagem de dois poços (BP-01 e BP-02) perfurados pela Petrobras S.A. na porção offshore da Bacia de Pelotas (Fig. 1) constituem o material estudado neste trabalho. Foram amostrados 535 níveis estratigráficos distintos, compreendendo idades do Cretáceo ao Neogeno.

0 tratamento para recuperação do conteúdo palinológico consistiu na maceração, eliminação da fração inorgânica por adição de reagentes ácidos e concentração da matéria orgânica particulada por peneiramento e montagem de lâminas. Essas etapas seguiram os procedimentos descritos por Uesugui (1979), resultando na confecção de 654 lâminas (para alguns níveis foram confeccio- 
nadas mais do que uma lâmina).

A análise taxonômica foi efetuada por meio da observação em microscópio óptico binocular ZEISS Imager.A2, em aumentos entre 200 e 1000 vezes e comparações com táxons descritos e ilustrados na literatura. Espécimes selecionados foram fotomicrografados com emprego de câmera digital AxioCam ICc3. A sistemática e disposição taxonômica utilizadas para a classificação dos cistos de dinoflagelados seguiram o ordenamento de Fensome et al. (1993) e Williams et al. (2017). Medições seguem o esquema apresentado na figura 3 , considerando os espécimes com melhor preservação, não refletindo, na maior parte dos casos, a quantidade absoluta de cada táxon. Descrições e ilustrações (Figs. 4 a 8) são apresentadas para todos os táxons; observações e comparações são oferecidas quando pertinentes.

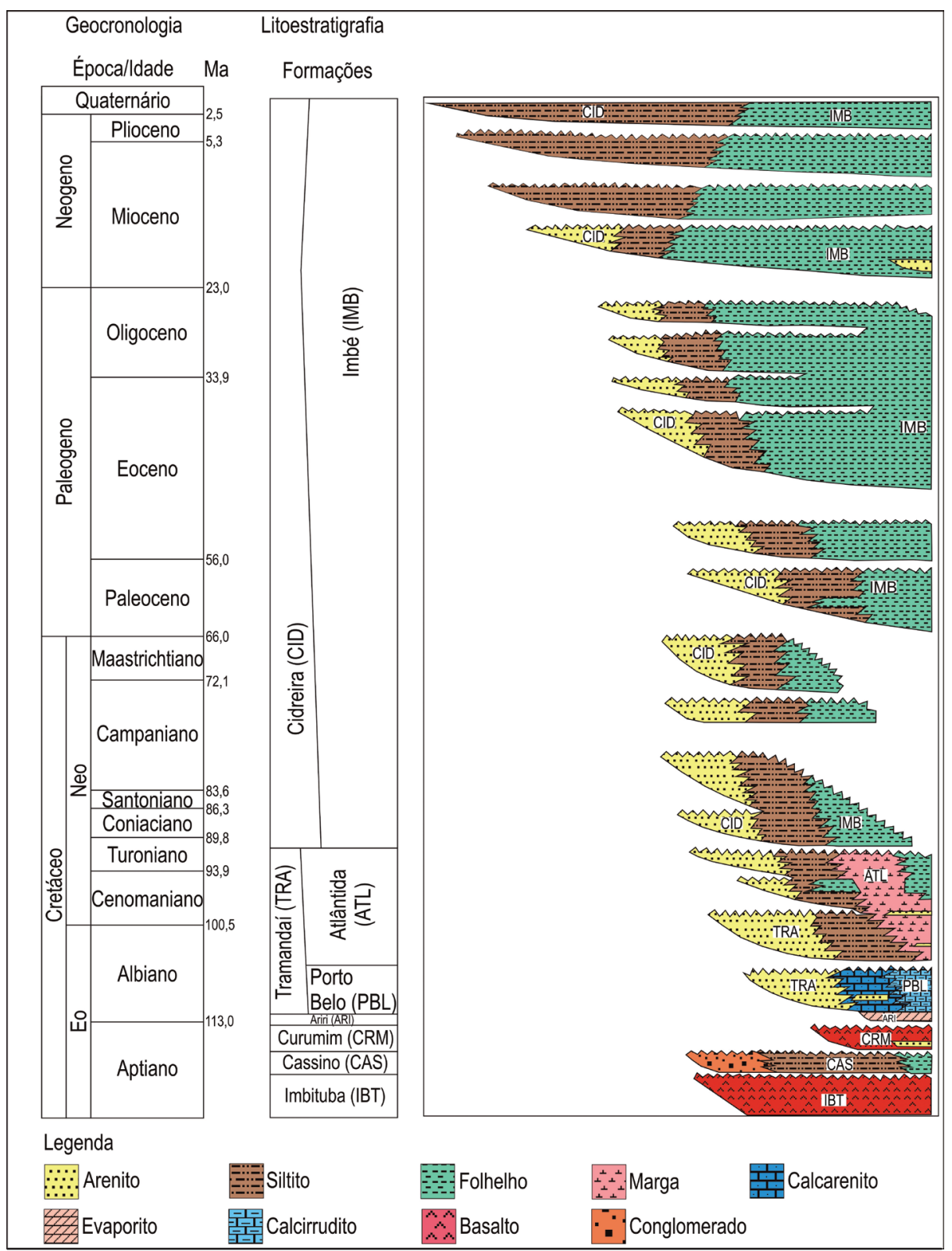

Figura 2. Carta estratigráfica simplificada da Bacia de Pelotas (modificado de Bueno et al., 2007; geocronologia conforme Gradstein et al., 2012).

Figure 2. Simplified stratigraphic chart of the Pelotas Basin (modified from Bueno et al., 2007; geochronology according to Gradstein et al., 2012).

\section{Resultados}

\subsection{Táxons identificados}

Os conjuntos palinológicos reconhecidos nos 535 níveis estudados são ricos e diversificados, constituídos por esporos (briófitas e pteridófitas), grãos de pólen (gimnospermas e angiospermas) e cistos de dinoflagelados; incluem ainda, em quantidades subordinadas, palinoforaminíferos, escolecodontes e esporos de fungos. Na maior parte dos níveis, os palinomorfos apresentam bom estado de preservação. 


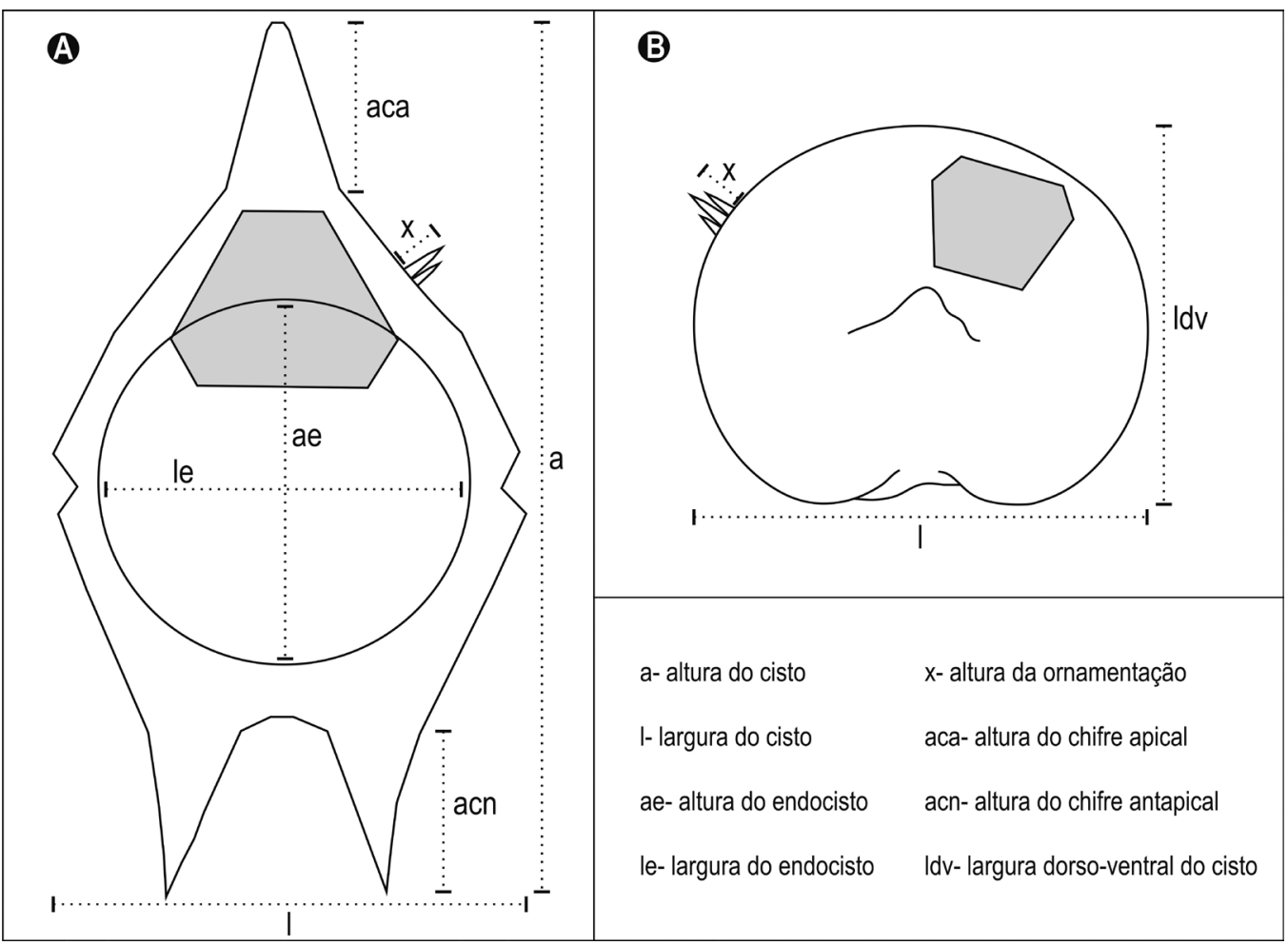

Figura 3. Esquemas de dois cistos de dinoflagelados peridinioides destacando parâmetros dimensionais e nomenclaturas utilizados neste trabalho (modificado de Evitt, 1985). A) Cisto com compressão dorso-ventral; B) Cisto com compressão apical-antapical.

Figure 3. Schematic sketch of two peridinian dinoflagellates cysts highlighting dimensional parameters and nomenclatures used in this paper (modified from Evitt, 1985). A) Cyst with dorso-ventral compression; B) Cyst with apical-antapical compression.

Dentre os 220 táxons de cistos de dinoflagelados reconhecidos, 76 são referentes à Ordem Peridiniales, dentre os quais 24 registrados pela primeira vez no Brasil: Cerodinium speciosum subsp. elongatum, Chatangiella granulifera, Deflandrea antarctica, D. convexa, D. denticulata, D. foveolata, D. oebisfeldensis, Lejeunecysta communis, L. pulchra, Manumiella seymourensis, Palaeocystodinium miocaenicum, Phthanoperidinium levimurum, $P$. multispinum, Petalodinium waipawaense, Piladinium columnum, Rhadinodinium glabrum, Selenopemphix conspicua, Senegalinium? dilwynense, Spinidinium? clavus, Spinidinium lanterna, Trinovantedinium harpagonium, T. variabile, Vozzhennikovia mariae e $V$. rotunda.

O levantamento sistemático e descritivo das associações de cistos peridinioides é apresentado a seguir, compreendendo 32 gêneros, 70 espécies e três subespécies. Dois táxons são descritos sem determinação específica: Barssidinium sp. 1 e Phelodinium sp. 1.

3.2 Sistemática paleontológica dos cistos de dinoflagelados peridinioides
Divisão Dinoflagellata (Butschli 1885) Fensome et al. 1993

Subdivisão Dinokaryota Fensome et al. 1993

Classe Dinophyceae Pascher 1914

Subclasse Peridiniphycidae Fensome et al. 1993

Ordem Peridiniales Haeckel 1894

Subordem Peridiniineae (Autonyum)

Família Peridiniaceae Ehrenberg 1831

Subfamília Palaeoperidinioidea (Vozzhennikova 1961) Burjak \& Davies 1983

Gênero Diconodinium Eisenack \& Cookson 1960 emend. Morgan 1977

Diconodinium lurense Guerstein et al. 2005

Fig. 4A-B

Descrição. Cisto proximado acavado de contorno fusiforme; chifres apical e antapical desenvolvidos; autofragma densamente coberto por espinhos; paracíngulo e parassulco distintos; arqueopilo intercalar tipo I (2a), sutura lateral e apical presente; opérculo preso; paratabulação indicada pelo paracíngulo e arqueopilo.

Dimensões (3 espécimes). a: 85-90 $\mu \mathrm{m}, \mathrm{l}: 42-55$ $\mu \mathrm{m}$; $\mathrm{x}$ (espinhos): 1-2 $\mu \mathrm{m}$; aca: 14-25 $\mu \mathrm{m}$; acn: 16$24 \mu \mathrm{m}$. 
Gênero Palaeoperidinium Deflandrea 1934 ex Sarjeant 1967 emend. Evitt et al. 1998

Palaeoperidinium pyrophorum (Ehrenberg 1838 ex Wetzel 1933) Sarjeant 1967 emend. Sarjeant 1967; Gocht \& Netzel 1976; Evitt, Damassa \& Albert 1998

Fig. 4C

Descrição. Cisto proximado cavado de contorno pentagonal com compressão dorso-ventral; epicisto e hipocisto simétricos; chifres relativamente curtos, um chifre apical, dois chifres antapicais simétricos; parede do cisto composta por endofragma, perifragma e exofragma; superfície parassutural e estriada; paracíngulo e parassulco distintos; arqueopilo combinado (A3I3P); opérculo livre, ocasionalmente preso; paratabulação indicada pelas feições parassuturais e arqueopilo.

Dimensões (3 espécimes). a: 118-127 $\mu \mathrm{m}$; l: 86$117 \mu \mathrm{m}$.

\section{Gênero Palaeohystrichophora Deflandre 1935} emend. Deflandre \& Cookson 1955

\section{Palaeohystrichophora infusorioides Deflandre 1935}

Fig. 4D

Descrição. Cisto próximo-condensado bicavado de contorno elipsoidal; um chifre apical, um chifre antapical; endocisto circular a oval finamente granulado; parede ornamentada por processos atabulares finos filiformes; paracíngulo distinto; parassulco indistinto; arqueopilo indistinto; paratabulação indicada somente pelo paracíngulo. Dimensões (1 espécime). Cisto, a: $68 \mu \mathrm{m}$; l: $61 \mu \mathrm{m}$; endocisto, ae: $38 \mu \mathrm{m}$; le: $44 \mu \mathrm{m}$; (processos): 6-8 $\mu \mathrm{m}$.

Gênero Phthanoperidinium Drugg \& Loeblich Jr. 1967 emend. Edwards \& Bebout 1981; Islam 1982

\section{Phthanoperidinium levimurum Bujak in Bujak et al. 1980}

Fig. 4E

Descrição. Cisto próximo-condensado de contorno subesférico; chifres apical e antapical pouco desenvolvidos; perifragma granulado a verrugado, superfície composta de cristas parassuturais finas, lisas ou onduladas; paracíngulo e parassulco distintos; arqueopilo tipo I (2a); opérculo livre; paratabulação indicada pelas feições parassuturais e arqueopilo.

Dimensões (1 espécime). a: $46 \mu \mathrm{m}$; l: $44 \mu \mathrm{m}$; $\mathrm{x}$ (cristas): 1,5-2 $\mu \mathrm{m}$.

Phthanoperidinium multispinum Bujak in Bujak et al. 1980

Fig. $4 \mathrm{~F}$

Descrição. Cisto próximo-condensado de contorno subesférico; chifres apical e antapical pouco desenvolvidos; perifragma liso a granulado, superfície composta de cristas parassuturais ornamentadas por pequenos espinhos; paracíngulo e parassulco distintos; arqueopilo tipo I (2a); opérculo livre; paratabulação indicada pelas feições parassuturais e arqueopilo.

Dimensões (1 espécime). a: $48 \mu \mathrm{m}$; l: $43 \mu \mathrm{m}$; x (espinhos): 2-3 $\mu \mathrm{m}$.

Subfamília Deflandreoideae Bujak \& Davies 1983

Gênero Amphidiadema (Cookson \& Eisenack 1960) Lentin \& Williams 1976

\section{Amphidiadema denticulata Cookson \& Eisenack 1960 \\ Fig. $4 \mathrm{H}$}

Descrição. Cisto proximado bicavado de contorno elipsoidal; extensão apical e antapical com superfície granular, extremidades sub-retangulares de bordas denticuladas; endocisto subesférico a elipsoidal de superfície lisa; paracíngulo e parassulco indistintos; arqueopilo intercalar tipo I (2a); paratabulação indicada somente pelo aqueopilo.

Dimensões ( 3 espécimes). Cisto, a: 59-75 $\mu \mathrm{m}$ l: $34-$ $38 \mu \mathrm{m}$; endocisto, ae: $37-49 \mu \mathrm{m}$; le: $34-38 \mu \mathrm{m}$.

Amphidiadema nucula (Cookson \& Eisenack 1962) Lentin \& Williams 1976

Fig. 4I

Descrição. Cisto proximado bicavado de contorno oval; extensão apical arredondada; extensão antapical truncada; superfície lisa; endocisto circular de superfície lisa; paracíngulo e parassulco indistintos; arqueopilo intercalar tipo I (2a); paratabulação indicada somente pelo arqueopilo.

Dimensões (1 espécime). Cisto, a: $56 \mu \mathrm{m}$; l: $44 \mu \mathrm{m}$; endocisto ae: $41 \mu \mathrm{m}$; le: $44 \mu \mathrm{m}$.

Gênero Andalusiella Riegel 1974 emend. Riegel \& Sarjeant 1982; Masure, Tea \& Yao 1996

Andalusiella dubia (Jain \& Millepied 1973) Lentin 
\& Williams 1980 emend. Masure, Tea \& Yao 1996 Fig. 4J

Descrição. Cisto proximado acrocavado de contorno subesférico com compressão dorso-ventral; chifres apical e antapicais pouco desenvolvidos, às vezes ausentes; perifragma liso; endofragma granulado; endocisto subesférico; paracíngulo distinto; parassulco indistinto; arqueopilo intercalar tipo I/I (2a) euri-deltaforme; opérculo livre ou preso; paratabulação indicada pelo paracíngulo e arqueopilo.

Dimensões (1 espécime). a: $92 \mu \mathrm{m}$; l: $87 \mu \mathrm{m}$.

\section{Andalusiella mauthei Riegel 1974, emend. Riegel \& Sarjeant 1982 \\ Fig. 4K-L}

Descrição: Cisto proximado acrocavado de contorno ovoidal a romboidal com compressão dorso-ventral; chifre apical curto com terminação cônica, dois chifres antapicais curtos e assimétricos com terminações cônicas; perifragma liso a granulado; endofragma liso; paracíngulo distinto; parassulco indistinto; arqueopilo intercalar tipo I (2a) iso-deltaforme; paratabulação indicada pelo paracíngulo e arqueopilo.

Dimensões (5 espécimes). Cisto, a: 120-141 $\mu \mathrm{m}$; : 69-89 $\mu \mathrm{m}$; endocisto, ae: 92-113 $\mu \mathrm{m}$; le: 69-89 $\mu \mathrm{m}$.

\section{Andalusiella rhomboides (Boltenhagen 1977) Len-} tin \& Williams 1980; Masure, Tea \& Yao 1996

Fig. 4M-N

Descrição. Cisto proximado acrocavado a cornucavado de contorno romboidal com compressão dorso-ventral; chifre apical curto com terminação cônica, chifre antapical curto com terminaçõe cônica; perifragma e endofragma grosseiramente granulado; endocisto subesférico a romboidal; paracíngulo distinto; parassulco indistinto; arqueopilo intercalar tipo I/I (2a) iso-deltaforme; opérculo livre, eventualmente preso; paratabulação indicada pelo paracíngulo e arqueopilo.

Dimensões (2 espécimes). Cisto, a: 118-139 $\mu \mathrm{m}$; l: 73-77 $\mu \mathrm{m}$; endocisto, ae: 84-97; le: 65-77 $\mu \mathrm{m}$.

Gênero Cerodinium Vozzhennikova 1963 emend. Lentin \& Williams 1987

Cerodinium boloniense (Riegel 1974) Lentin \& Williams 1989 emend. Riegel \& Sarjeant 1982 Fig. 40-P

Descrição. Cisto proximado circuncavado a cornu- cavado de contorno pentagonal com compressão dorso-ventral; chifre apical desenvolvido; chifres antapicais desenvolvidos e simétricos; endocisto subesférico a pentagonal; perifragma liso apresentando estrias e dobras dispostas verticalmente; paracíngulo distinto; parassulco indicado por uma leve depressão; arqueopilo intercalar tipo I (2a); opérculo livre; paratabulação indicada pelo paracíngulo, parassulco e arqueopilo.

Dimensões (3 espécimes). Cisto, a: 91-105 $\mu \mathrm{m}$; l: 68-76 $\mu \mathrm{m}$; endocisto, ae: $61-73 \mu \mathrm{m}$; le: $68-76 \mu \mathrm{m}$.

\section{Cerodinium dartmoorium (Cookson \& Eisenack 1965) Lentin \& Williams 1987}

Fig. 4Q-R

Descrição. Cisto proximado bicavado com compressão dorso-ventral; chifre apical relativamente longo; chifres antapicais simétricos; epicisto maior que o hipocisto; endocisto circular a oval; perifragma com características paratabulares, ornamentação composta por espinhos intratabulares e parassuturais; paracíngulo e parassulco distintos; arqueopilo intercalar tipo I (2a) iso-deltaforme; opérculo livre; paratabulação indicada pelas feições parassuturais e arqueopilo.

Dimensões (2 espécimes). Cisto, a: 114-126 $\mu \mathrm{m}$; l:

71-75 $\mu \mathrm{m}$; endocisto, ae: 61-63 $\mu \mathrm{m}$; le: $63-71 \mu \mathrm{m}$; $\mathrm{x}$ (espinhos): 1-1,5 $\mu \mathrm{m}$.

\section{Cerodinium speciosum (Alberti 1959) Lentin \& Williams 1987}

Fig. $4 \mathrm{~S}$

Descrição. Cisto proximado bicavado com compressão dorso-ventral; chifre apical longo; chifres antapicais longos e simétricos; endocisto circular a oval; perifragma ornamentado por pequenos grânulos e espinhos moderadamente alinhados; paracíngulo distinto; parassulco indistinto; arqueopilo tipo I (2a) iso-deltaforme; opérculo livre; paratabulação indicada pelo paracíngulo e arqueopilo.

Dimensões (4 espécimes). Cisto, a: 111-153 $\mu \mathrm{m}$; l: 82-93 $\mu \mathrm{m}$; endocisto, ae: $58-66 \mu \mathrm{m}$; le: $82-93 \mu \mathrm{m}$; $\mathrm{x}$ (espinhos): $1 \mu \mathrm{m}$.

Cerodinium speciosum subsp. elongatum (Mao Shaozhi \& Norris 1988) Lentin \& Williams 1989 Fig. $4 \mathrm{~T}$

Descrição. Cisto proximado circuncavado com compressão dorso-ventral; epicisto maior que o hipocisto; chifre apical longo; chifres antapicais simétricos; endocisto circular a oval; perifragma ornamentado por pequenos grânulos e espinhos 
moderadamente alinhados; paracíngulo distinto; parassulco indistinto; arqueopilo tipo I (2a) iso-deltaforme; opérculo livre; paratabulação indicada pelo paracíngulo e arqueopilo.

Dimensões (1 espécime). Cisto, a: $152 \mu \mathrm{m}$; l: 81 $\mu \mathrm{m}$; endocisto ae: $82 \mu \mathrm{m}$; le: $81 \mu \mathrm{m}$ de largura; $\mathrm{x}$ (espinhos): 1-2 $\mu \mathrm{m}$.

\section{Cerodinium striatum (Drugg 1967) Lentin \& Williams 1987}

Fig. $4 \mathrm{U}$

Descrição. Cisto proximado bicavado com compressão dorso-ventral; chifre apical longo; chifres antapicais simétricos; perifragma ornamentado por dobras paralelas dispostas longitudinalmente ao longo do pericisto; endocisto circular a oval; paracíngulo distinto; parassulco indistinto; arqueopilo intercalar tipo I (2a) iso-deltaforme; opérculo livre; paratabulação indicada pelo paracíngulo e arqueopilo.

Dimensões (2 espécimes). Cisto, a: 137-156 $\mu \mathrm{m}$; l: 73-94 $\mu \mathrm{m}$; endocisto, ae: 66-80 $\mu \mathrm{m}$; le: 73-87 $\mu \mathrm{m}$.

\section{Gênero Chatangiella Vozzhennikova 1967}

\section{Chatangiella? biapertura (McIntyre 1975) Lentin \& Williams 1976}

Fig. $4 \mathrm{~V}$

Descrição. Cisto proximado bicavado com compressão dorso-ventral; chifre apical curto de terminação acuminada; ombros não proeminentes; chifres antapicais assimétricos, sendo o esquerdo maior com terminações cônicas; endocisto oval (mais largo do que comprido); endofragma liso; perifragma finamente granulado; parassulco indistinto, paracíngulo bem marcado e não contínuo; arqueopilo intercalar tipo I (2a); opérculo livre; paratabulação indicada somente pelo paracíngulo e arqueopilo.

Dimensões (5 espécimes). Cisto, a: $102-128 \mu \mathrm{m}$; l: 61-79 $\mu \mathrm{m}$; endocisto, ae: 40-77 $\mu \mathrm{m}$; le: $60-73 \mu \mathrm{m}$.

\section{Chatangiella granulifera (Manum 1963) Lentin \& Williams 1976}

Fig. 5A

Descrição. Cisto proximado bicavado com compressão dorso-ventral; chifre apical curto de terminação cônica; ombros proeminentes; chifres antapicais assimétricos, sendo o direito maior com terminações cônicas; endocisto circular; pe- rifragma e endofragma fortemente granulado; parassulco indistinto, paracíngulo bem marcado e não continuo contínuo; arqueopilo intercalar tipo I (2a); endoaqueopilo tipo 3I; opérculo livre; paratabulação indicada somente pelo paracíngulo e arqueopilo.

Dimensões (1 espécime). Cisto, a: $107 \mu \mathrm{m}$; l: 70 $\mu \mathrm{m}$; endocisto, ae: $55 \mu \mathrm{m}$; le: $70 \mu \mathrm{m}$.

Chatangiella spectabilis (Alberti 1959) Lentin \& Williams 1976 emend. Lebedeva in Ilyina, Kulkova \& Lebedeva 1994

Fig. 5B

Descrição. Cisto proximado bicavado com compressão dorso-ventral; chifre apical curto de terminação cônica; ombros proeminentes; chifres antapicais assimétricos, sendo o maior com terminações cônicas; endocisto circular; endofragma liso, perifragma finamente granulado; parassulco indistinto, paracíngulo bem marcado e contínuo; arqueopilo intercalar tipo I (2a); opérculo livre; paratabulação indicada somente pelo paracíngulo e arqueopilo.

Dimensões (3 espécimes). Cisto, a: 102-147 $\mu \mathrm{m}$; l: 60-80 $\mu \mathrm{m}$; endocisto, ae: $40-80 \mu \mathrm{m}$; le: $66-100 \mu \mathrm{m}$. Comparações. Chatangiella spectabilis difere-se de Chatangiella? biapertura por apresentar ombros proeminentes e um paracíngulo contínuo.

Gênero Deflandrea Eisenack 1938 emend. Williams \& Downie 1966; Stover 1974; Lentin \& Williams 1976

\section{Deflandrea antarctica Wilson 1967 Fig. 5C-D}

Descrição. Cisto proximado bicavado com compressão dorso-ventral; chifre apical de terminação cônica; chifres antapicais curtos e simétricos; perifragma granulado; endocisto circular e liso; parassulco indistinto, paracíngulo bem marcado e contínuo; periarqueopilo intercalar tipo I/I (2a) iso-deltaforme a lati-deltaforme; endoarqueopilo lati-deltaforme; opérculo livre; paratabulação indicada somente pelo paracíngulo e arqueopilo.

Dimensões (4 espécimes). Cisto, a: $110-129 \mu \mathrm{m}$; $\mathrm{l}$ 79-97 $\mu \mathrm{m}$; endocisto, ae: 73-80 $\mu \mathrm{m}$; le: 70-78 $\mu \mathrm{m}$.

\section{Deflandrea convexa Wilson 1988}

Fig. 5E-F 


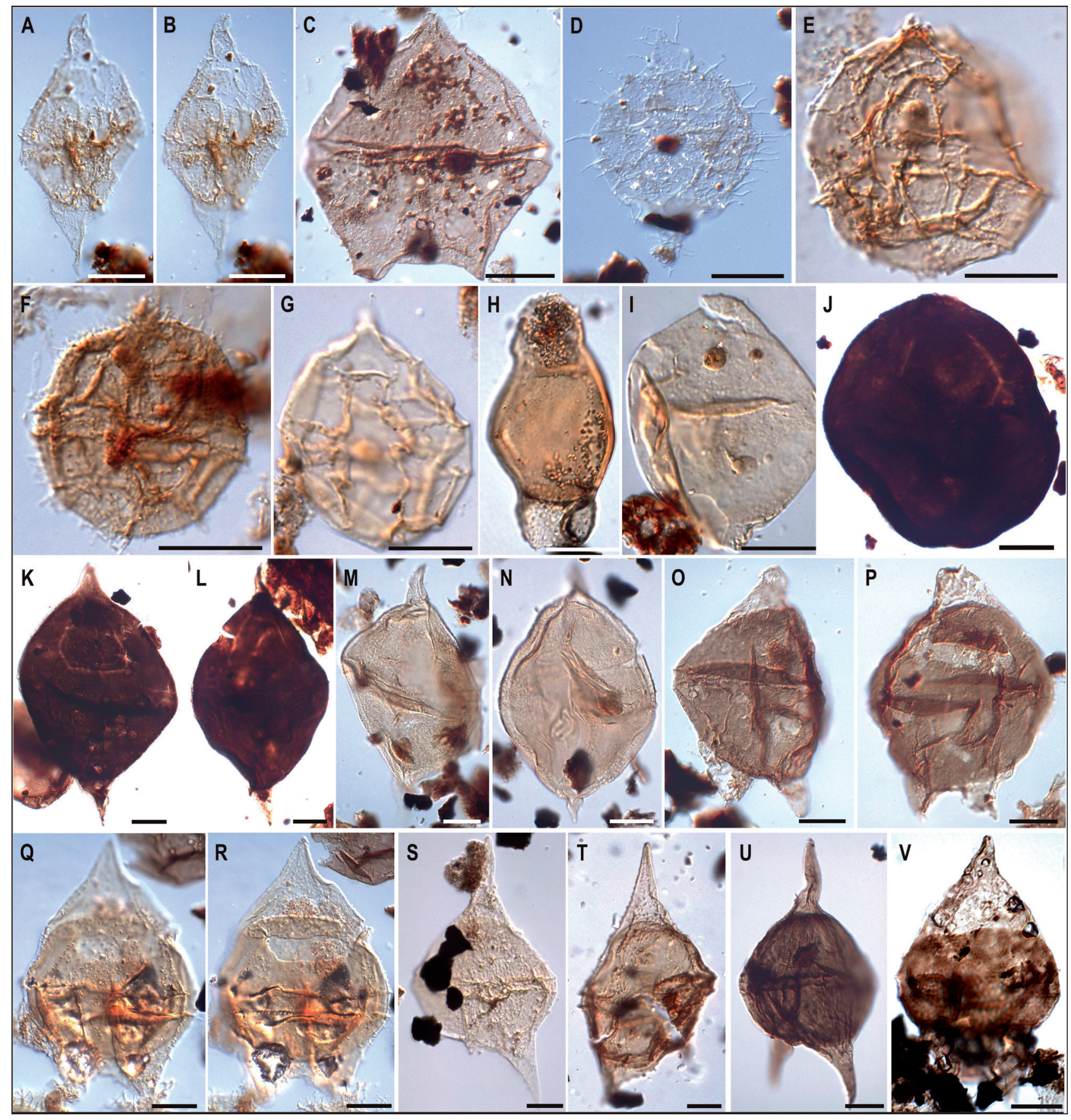

Figura 4. Fotomicrografias de cistos de dinoflagelados peridinioides selecionados (escala gráfica equivalente a $20 \mu \mathrm{m}$ ). A-B) Diconodinium lurense (poço BP-02, profundidade 3.791,55 m, coordenada England Finder U36-3); C) Palaeoperidinium pyrophorum (BP-01, 3.840-3.855 m, H43-3); D) Palaeohystrichophora infusorioides (BP-02, 5.070-5.085 m, P44-4); E) Phthanoperidinium levimurum (BP-02, 2.025-2.040 m, E31-1); F) Phthanoperidinium multispinum (BP-01, 1.303,10 m, Q29); G) Phthanoperidinium sp. (BP-01, 3.058,54 m, J39-1); H) Amphidiadema denticulata (BP-02, 4.170-4.185 m, M38-2); I) Amphidiadema nucula (BP-02, 4.380-4.395 m, L47-2); J) Andalusiella dubia (BP-02, 3.790,37 m, P33); K-L) Andalusiella mauthei (K: BP-02, $3.790,37$ m, G39-4, L: BP-02, 3.790,37 m, D32); M-N) Andalusiella rhomboides (M: BP-02, 3.786-3.795 m, X41-3, N: BP-02, 3.786-3.795 m, L33-2); O-P) Cerodinium boloniense (O: BP-02, 3.790,37 m, F37-3, P: BP-02, 3.790,37 m, C36-2); Q-R) Cerodinium dartmoorium (BP-01, 3.057,98 m, R37); S) Cerodinium speciosum (BP-02, 3.120-3.135 m, Q39-4); T) Cerodinium speciosum subsp. elongatum (BP-02, 3.090-3.105 m, N45-3); U) Cerodinium striatum (BP-01, 3.792,78 m, E35-1); V) Chatangiella? biapertura (BP-01, 4.486,62 m, H44-4).

Figure 4. Photomicrographs of selected peridinian dinoflagellate cysts (graphic scale equivalents to $20 \mu \mathrm{m}$ ).

Descrição. Cisto proximado bicavado com compressão dorso-ventral; ápice e antápice arredondados, sem chifres; pericoel desenvolvido somente no ápice e antápice; perifragma finamente reticulado; endocisto circular e liso; paracíngulo e parassulco indistintos; periarqueopilo intercalar tipo I (2a) iso-deltaforme a lati-deltaforme; endoarqueopilo lati-deltaforme; opérculo livre; paratabulação indicada somente pelo arqueopilo.

Dimensões (1 espécime). Cisto, a: $73 \mu \mathrm{m}$; l: $60 \mu \mathrm{m}$; endocisto, ae: $59 \mu \mathrm{m}$; le: $60 \mu \mathrm{m}$. 


\section{Deflandrea denticulata Alberti 1959}

Fig. 5G

Descrição. Cisto proximado bicavado de contorno pentagonal com compressão dorso-ventral; chifres apical e antapicais longos; perifragma coberto por pequenos espinhos; endocisto de forma pentagonal; paracíngulo distinto; parassulco indistinto, arqueopilo intercalar tipo I (2a); opérculo livre; paratabulação indicada somente pelo paracíngulo e arqueopilo.

Dimensões (1 espécime). Cisto, a: $121 \mu \mathrm{m}$; l: 80 $\mu \mathrm{m}$; endocisto, ae: $58 \mu \mathrm{m}$; le: $80 \mu \mathrm{m}$; (espinhos): 1-2 $\mu \mathrm{m}$.

\section{Deflandrea foveolata Wilson 1984}

Fig. 5H-J

Descrição. Cisto proximado bicavado com compressão dorso-ventral; chifres apical e antapicais desenvolvidos, com pequena estrutura sólida nas extremidades distais; perifragma fino; endocisto circular de parede espessa, superfície foveolada; paracíngulo e parassulco distinto, periarqueopilo intercalar tipo I; endoarqueopilo lati-deltaforme; opérculo livre; paratabulação indicada somente pelo paracíngulo e arqueopilo.

Dimensões (4 espécimes). Cisto, a: $128-149 \mu \mathrm{m}$; l: 90-99 $\mu \mathrm{m}$; endocisto, ae: 73-81 $\mu \mathrm{m}$; le: $90-99 \mu \mathrm{m}$.

\section{Deflandrea heterophlycta Deflandre \& Cookson 1955}

Fig. 5K

Descrição. Cisto proximado bicavado com compressão dorso-ventral; chifre apical de terminação cônica; chifres antapicais simétricos; perifragma granulado; endocisto circular e granulado, ornamentdo com projeções semelhantes a tubérculos relativamente grandes; paracíngulo distinto; parassulco indistinto, periarqueopilo intercalar tipo I/I (2a) iso-deltaforme a lati-deltaforme; endoarqueopilo lati-deltaforme; opérculo livre; paratabulação indicada somente pelo paracíngulo e arqueopilo.

Dimensões (3 espécimes). Cisto, a: 100-117 $\mu \mathrm{m}$; l: 79-93 $\mu \mathrm{m}$; endocisto, ae: $65-83 \mu \mathrm{m}$; le: $79-88 \mu \mathrm{m}$; (tubérculos): 1-3 $\mu \mathrm{m}$.

\section{Deflandrea oebisfeldensis Alberti 1959} Fig. 5L

Descrição. Cisto proximado circuncavado a bicavado com compressão dorso-ventral; epicisto geralmente maior que o hipocisto; chifre apical desen- volvido de terminação cônica; chifres antapicais relativamente curtos e simétricos; pericoel antapical desenvolvido; perifragma granulado; endocisto circular a subésferico; paracíngulo bem marcado e contínuo; parassulco indicado por uma leve depressão; periarqueopilo intercalar tipo I/I (2a) iso-deltaforme a lati-deltaforme; endoarqueopilo lati-deltaforme; opérculo livre; paratabulação indicada somente pelo paracíngulo e arqueopilo.

Dimensões (6 espécimes). Cisto, a: 125-144 $\mu \mathrm{m}$; l: 84-112 $\mu \mathrm{m}$; endocisto, ae: 61-73 $\mu \mathrm{m}$; le: 65-90 $\mu \mathrm{m}$.

\section{Deflandrea phosphoritica Eisenack 1938} Fig. 5M-N

Descrição. Cisto proximado bicavado a circuncavado com compressão dorso-ventral; epicisto geralmente menor que o hipocisto; chifre apical curto de terminação cônica; chifres antapicais curtos e simétricos; perifragma finamente granulado; endocisto circular e finamente granulado; paracíngulo e parassulco distintos; periarqueopilo intercalar tipo I/I (2a) iso-deltaforme a lati-deltaforme; endoarqueopilo lati-deltaforme; opérculo livre; paratabulação indicada pelo paracíngulo, parassulco e arqueopilo.

Dimensões (2 espécimes). Cisto, a: 102-113 $\mu \mathrm{m}$; l: 75-80 $\mu \mathrm{m}$; endocisto, ae: 57-61 $\mu \mathrm{m}$; le: 75-80 $\mu \mathrm{m}$.

\section{Deflandrea robusta Deflandre \& Cookson 1955} Fig. 50-P

Descrição. Cisto proximado bicavado com compressão dorso-ventral; chifre apical de terminação cônica; chifres antapicais curtos e ligeiramente assimétricos; perifragma liso, mas pequenos grânulos podem estar presentes no epicisto; endocisto subesférico e liso; parassulco e paracíngulo fracamente marcados; arqueopilo intercalar tipo I (2a) iso-deltaforme a lati-deltaforme; opérculo livre; paratabulação indicada somente pelo paracíngulo e arqueopilo.

Dimensões (3 espécimes). Cisto, a: 110-117 $\mu \mathrm{m}$; l: 66-78 $\mu \mathrm{m}$; endocisto, ae: 59-68 $\mu \mathrm{m}$; le: 66-78 $\mu \mathrm{m}$.

Gênero Isabelidinium Lentin \& Williams 1977 emend. Marshall 1988

Isabelidinium cretaceum (Cookson 1956) Lentin \& Williams 1977

Fig. 5 Q

Descrição. Cisto bicavado com compressão dorso-ventral; chifre apical curto pouco desenvolvido; ombros pouco desenvolvidos; chifres antapicais 
assimétricos, sendo o esquerdo mais desenvolvido; endocisto oval (mais largo do que comprido); endofragma liso; perifragma finamente granulado; parassulco e paracíngulo indistintos; arqueopilo intercalar tipo I (2a) lati-tetaforme; opérculo livre; paratabulação indicada somente pelo arqueopilo. Dimensões (3 espécimes). Cisto, a: 71-100 $\mu \mathrm{m}$; l: 55-76 $\mu \mathrm{m}$; endocisto, ae: 22-69 $\mu \mathrm{m}$; le: 50-76 $\mu \mathrm{m}$.

\section{Isabelidinium glabrum (Cookson \& Eisenack 1969) Lentin \& Williams 1977}

Fig. 5R

Descrição. Cisto proximado bicavado com compressão dorso-ventral; chifre apical curto de terminação acuminada; ombros pouco proeminentes; chifres antapicais assimétricos, sendo esquerdo maior com terminações cônicas; endocisto oval a circular; endofragma liso; perifragma liso; parassulco e paracíngulo indistintos; arqueopilo intercalar tipo I (2a) iso-deltaforme; opérculo preso; paratabulação indicada somente pelo arqueopilo. Dimensões (2 espécimes). Cisto, a: $120-130 \mu \mathrm{m}$; l: 70-78 $\mu \mathrm{m}$; endocisto, ae: 55-62 $\mu \mathrm{m}$; le: 70-78 $\mu \mathrm{m}$.

\section{Isabelidinium korojonense (Cookson \& Eisenack} 1958) Lentin \& Williams 1977

Fig. 5S-T

Descrição. Cisto bicavado com compressão dorso-ventral; chifre apical pouco desenvolvido; ombros bem desenvolvidos; chifres antapicais com as extremidades pouco desenvolvidas; endocisto oval (mais largo do que comprido); endofragma e perifragma finamente granulados; parassulco e paracíngulo indistintos; arqueopilo intercalar tipo I (2a) iso-omegaforme; opérculo livre; paratabulação indicada somente pelo arqueopilo.

Dimensões (5 espécimes). Cisto, a: 69-85 $\mu \mathrm{m}$; l: 51$60 \mu \mathrm{m}$; endocisto, ae: 29-38 $\mu \mathrm{m}$; le: 52-65 $\mu \mathrm{m}$.

\section{Isabelidinium pellucidum (Deflande \& Cookson 1955) Lentin \& Williams 1977}

Fig. $5 \mathrm{U}$

Descrição. Cisto proximado bicavado com compressão dorso-ventral; chifre apical curto de terminação acuminada; ombros pouco proeminentes; chifres antapicais assimétricos, sendo esquerdo maior com terminações cônicas; endocisto oval (geralmente mais largo do que comprido) a circular; endofragma liso; perifragma finamente granulado; parassulco e paracíngulo indistintos; arqueo- pilo intercalar tipo I (2a) iso-deltaforme; opérculo livre; paratabulação indicada somente pelo arqueopilo.

Dimensões (5 espécimes). Cisto, a: $102-133 \mu \mathrm{m}$; l: 65-89 $\mu \mathrm{m}$; endocisto, ae: 55-65 $\mu \mathrm{m}$; le: $62-89 \mu \mathrm{m}$.

Comparações. Isabelidinium pellucidum difere de outras espécies do gênero por possuir um perifragma finamente granulado e ombros pouco proeminentes.

Isabelidinium? viborgense Heilmann-Clausen 1985 Fig. 5V-W

Descrição. Cisto proximado circuncavado de contorno pentagonal com compressão dorso-ventral; epicisto e hipocisto simétricos; chifre apical curto; chifres antapicais curtos; endocisto subesférico; perifragma fino e liso; paracíngulo e parassulco distintos; arqueopilo intercalar tipo I (2a), podendo ocorrer também em combinação intercalar com pré-cingular; opérculo livre; paratabulação indicada pelo paracíngulo, parassulco e arqueopilo.

Dimensões ( 5 espécimes). Cisto, a: 45-56 $\mu \mathrm{m}$; l: 35$42 \mu \mathrm{m}$; endocisto, ae: $29-33 \mu \mathrm{m}$; le: $35-42 \mu \mathrm{m}$.

Comparações. Isabelidinium? viborgense difere de outras espécies do gênero por possuir uma marcante depressão na região do paracíngulo; o parassulco é bem desenvolvido, estendendo-se até a extremidade antapical.

\section{Gênero Manumiella Bujak \& Davies 1983}

Manumiella seelandica (Lange 1969) Bujak \& Davies 1983 emend. Firth 1987

Fig. 6A

Descrição. Cisto proximado circuncavado de contorno romboidal com compressão dorso-ventral; epicisto e hipocisto levemente arredondados; chifre apical curto; chifre antapical curto; perifragma finamente granulado; endocisto relativamente grande de contorno oval a circular, parede lisa a finamente escabrada; parassulco e paracíngulo indistintos; arqueopilo intercalar tipo I (2a); opérculo livre; paratabulação indicada somente pelo arqueopilo.

Dimensões (2 espécimes). Cisto, a: 83-86 $\mu \mathrm{m}$; l: $67-$ $74 \mu \mathrm{m}$; endocisto, ae: $58-61 \mu \mathrm{m}$; le: 58-59 $\mu \mathrm{m}$.

\section{Manumiella seymourensis Askin 1999}

Fig. 6B-C 


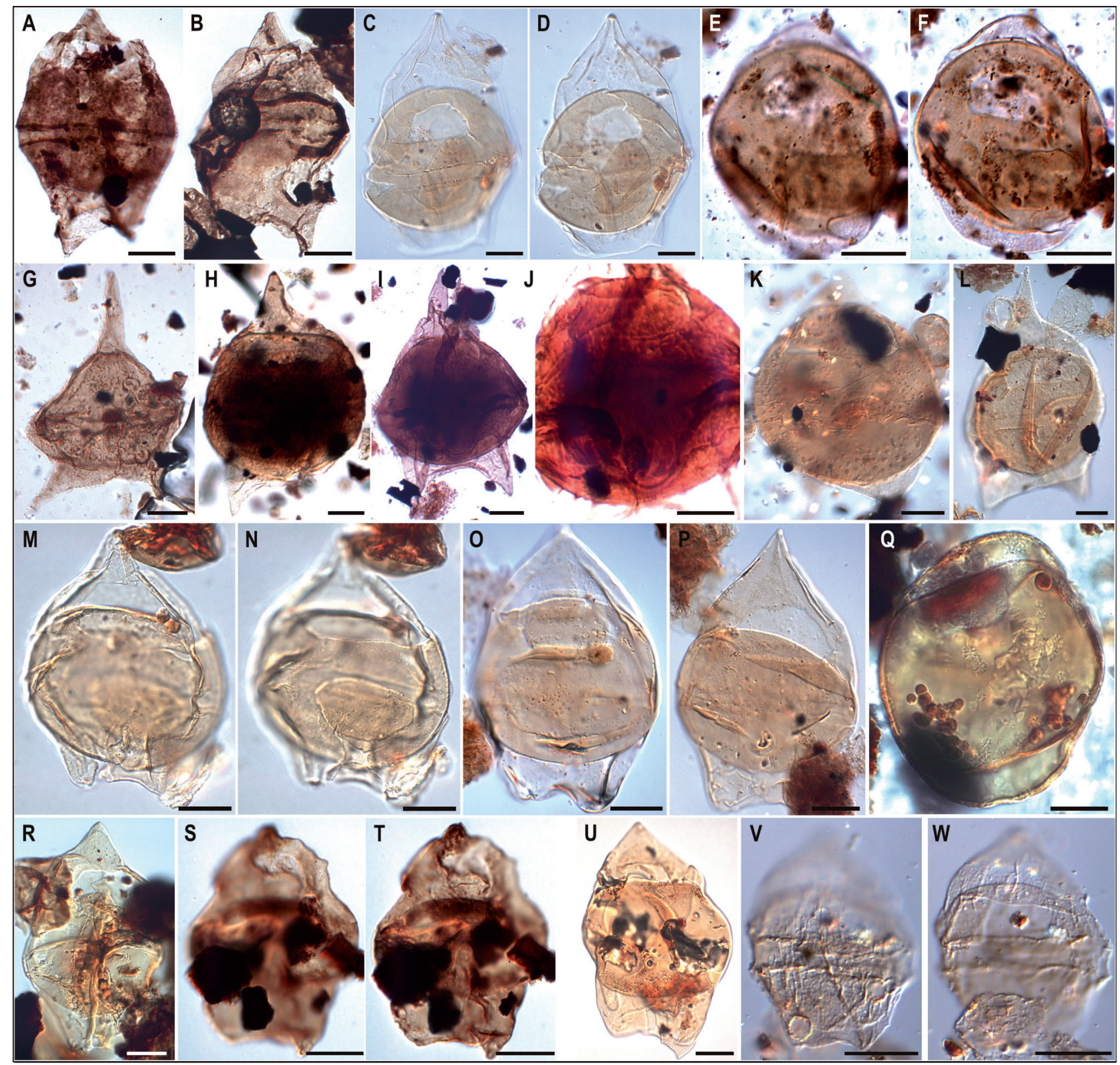

Figura 5. Fotomicrografias de cistos de dinoflagelados peridinioides selecionados (escala gráfica equivalente a $20 \mu \mathrm{m}$ ). A) Chatangiella granulifera (poço BP-01, profundidade 4.485,56 m, coordenada England Finder G48-4); B) Chatangiella spectabilis (BP-01, 4.484,80 m, E40-4); C-D) Deflandrea antarctica (BP-01, 2.540,35 m, S31); E-F) Deflandrea convexa (BP-01, 2.940-2.955 m, Q41); G) Deflandrea denticulata (BP-01, 3.810-3.825 m, L34-2); H-J) Deflandrea foveolata (H: BP-02, 3.180-3.195 m, N31-3, I-J: BP-01, 3.540-3.555 m, A36); K) Deflandrea heterophlycta (BP-01, 3.990-3.405 m, P43-4); L) Deflandrea oebisfeldensis (BP02, 2.932,25 m, R39-1); M-N) Deflandrea phosphoritica (BP-01, 2.539,43 m, K47); O-P) Deflandrea robusta (0: BP-01, $3.056,75$ m, E44, P: BP-01, 3.056,75 m, S42); Q) Isabelidinium cretaceum (BP-02, 3.420-3.435 m, P43-4); R) Isabelidinium glabrum (BP02, 3.790,37 m, L44); S-T) Isabelidinium korojonense (BP-01, 4.484,80 m, U39-4); U) Isabelidinium pellucidum (BP-02, $3.794,29$ $\mathrm{m}, \mathrm{T} 36-4) ; \mathrm{V}-\mathrm{W}$ ) Isabelidinium? viborgense (BP-02, 3.787-3.796 m, V33).

Figure 5. Photomicrographs of selected peridinian dinoflagellate cysts (graphic scale equivalents to $20 \mu \mathrm{m}$ ).

Descrição. Cisto proximado circuncavado de contorno oval com compressão dorso-ventral; epicisto e hipocisto arredondados, pequena saliência presente; perifragma fino, liso ou finamente granulado; endocisto subcircular, parede fina e lisa, endocisto às vezes pouco visível; parassulco e paracíngulo indistintos; arqueopilo intercalar tipo I (2a); opérculo livre; paratabulação indicada somente pelo arqueopilo.

Dimensões (2 espécimes). Cisto, a: 76-84 $\mu \mathrm{m}$; 1: 60$62 \mu \mathrm{m}$; endocisto, ae: $47-49 \mu \mathrm{m}$; le: $44-47 \mu \mathrm{m}$.

\section{Gênero Nelsoniella Cookson \& Eisenack 1960}

\section{Nelsoniella aceras Cookson \& Eisenack 1960} Fig. 6D

Descrição. Cisto proximado epicavado de contorno subesférico com compressão dorso-ventral; epicisto ornamentado por grânulos; hipocisto liso; endocisto subesférico a lenticular com parade lisa a finamente granulada; parassulco e paracíngulo indistintos; arqueopilo intercalar tipo I (2a); opér- 
culo livre; paratabulação indicada somente pelo arqueopilo.

Dimensões (2 espécimes). Cisto, a: 79-95 $\mu \mathrm{m}$; 1: 79$95 \mu \mathrm{m}$; endocisto, ae: $48-53 \mu \mathrm{m}$; le: 79-95 $\mu \mathrm{m}$.

Nelsoniella tuberculata Cookson \& Eisenack 1960 Fig. 6E

Descrição. Cisto proximado epicavado de contorno subesférico com compressão dorso-ventral; chifre apical pequeno; epicisto ornamentado por pequenos tubérculos ou grânulos; hipocisto liso; endocisto subesférico a lenticular com parade lisa a finamente granulada; parassulco e paracíngulo indistintos; arqueopilo intercalar tipo I (2a); opérculo livre; paratabulação indicada somente pelo arqueopilo.

Dimensões ( 1 espécime). Cisto, a: $63 \mu \mathrm{m}$; l: $61 \mu \mathrm{m}$; endocisto, ae: $38 \mu \mathrm{m}$; le: $61 \mu \mathrm{m}$.

\section{Gênero Paleocystodinium Alberti 1961}

\section{Palaeocystodinium australinum (Cookson 1965) Lentin \& Williams 1976 \\ Fig. $6 \mathrm{~F}$}

Descrição. Cisto proximado cornucavado a circuncavado de contorno fusiforme; chifre apical e antapical longos, chifre antapical acessório comumente presente; nenhuma característica parassutural, parede lisa ou ligeiramente granulada; paracíngulo e parassulco indistintos; arqueopilo intercalar tipo I (2a); paratabulação indicada pelo arqueopilo. Dimensões (4 espécimes). Cisto, a: 174-224 $\mu \mathrm{m}$; l: 44-51 $\mu \mathrm{m}$; endocisto, ae: $72-116 \mu \mathrm{m}$; le: $44-51 \mu \mathrm{m}$.

\section{Palaeocystodinium bulliforme Ioannides 1986} Fig. 6G

Descrição. Cisto proximado cornucavado a circuncavado de contorno fusiforme; chifre apical e antapical longos apresentando extremidades acuminadas a arredondadas; endocisto caracteristicamente túrgido; nenhuma característica parassutural, parede lisa a finamente granulada; paracíngulo e parassulco indistintos; arqueopilo intercalar tipo I (2a); opérculo livre; paratabulação indicada pelo arqueopilo.

Dimensões ( 2 espécimes). Cisto, a: 143-171 $\mu \mathrm{m}$; l: 53-61 $\mu \mathrm{m}$; endocisto, ae: 63-86 $\mu \mathrm{m}$; le: 53-61 $\mu \mathrm{m}$.

\section{Palaeocystodinium golzowense Alberti 1961 Fig. $6 \mathrm{H}$}

Descrição. Cisto proximado cavado, de contorno fusiforme; chifre apical e antapical longos e marcadamente afinados distalmente; nenhuma característica parassutural, parede lisa e fina; paracíngulo e parassulco indistintos; arqueopilo intercalar tipo I (2a); opérculo livre; paratabulação indicada pelo arqueopilo.

Dimensões (1 espécime). Cisto, a: $151 \mu \mathrm{m}$; l: 29 $\mu \mathrm{m}$; endocisto, ae: $59 \mu \mathrm{m}$; le: $29 \mu \mathrm{m}$.

\section{Palaeocystodinium lidiae (Górka 1963) Davey 1969 \\ Fig. 6I}

Descrição. Cisto proximado cavado, de contorno fusiforme; chifres apical e antapical longos, espessamento longitudinal interior ao longo de cada chifre; nenhuma característica parassutural, parede lisa; paracíngulo e parassulco indistintos; arqueopilo intercalar tipo I (2a); opérculo livre; paratabulação indicada pelo arqueopilo.

Dimensões (4 espécimes). Cisto, a: 165-223 $\mu \mathrm{m}$; l: 36-46 $\mu \mathrm{m}$; endocisto, ae: 80-125 $\mu \mathrm{m}$; le: $36-46 \mu \mathrm{m}$.

\section{Palaeocystodinium miocaenicum Strauss in Strauss, Lund \& Lund-Christensen 2001}

Fig. 6J

Descrição. Cisto proximado cornucavado de contorno fusiforme; chifre apical e antapical longos, extremidades distais dos chifres ornamentadas por pequenos grânulos; parede lisa exceto nas extreminades dos chifres; paracíngulo e parassulco indistintos; arqueopilo intercalar tipo I (2a); opérculo livre; paratabulação indicada pelo arqueopilo. Dimensões (2 espécimes). Cisto, a: 130-156 $\mu \mathrm{m}$; l: 26-27 $\mu \mathrm{m}$; endocisto, ae: 58-60 $\mu \mathrm{m}$; le: $26-27 \mu \mathrm{m}$.

\section{Gênero Pierceites Habib \& Drugg 1987}

\section{Pierceites pentagonus (May 1980) Habib \& Drugg 1987}

Fig. 6K-L

Descrição. Cisto proximado acavado de contorno pentagonal com compressão dorso-ventral; chifre apical curto de terminação acuminada; chifres antapicais assimétricos; parede fina e lisa; parassulco e paracíngulo indistintos; arqueopilo intercalar composto tipo 3I; opérculo livre; paratabulação indicada pelo arqueopilo.

Dimensões (5 espécimes). a: 74-92 $\mu \mathrm{m}$; l: 56-72 $\mu \mathrm{m}$.

Pierceites schizocystis Habib \& Drugg 1987 Fig. 6M 
Descrição. Cisto proximado acavado de contorno arredondado com compressão dorso-ventral; chifre apical curto de terminação acuminada; chifres antapicais assimétricos; parede fina, escabrada ou finamente granulada; parassulco e paracíngulo indistintos; arqueopilo intercalar composto tipo 3I; opérculo livre; paratabulação indicada pelo arqueopilo.

Dimensões (3 espécimes). a: 65-74 $\mu$ m; l: 45-60 $\mu \mathrm{m}$.

Gênero Senegalinium Jain \& Millepied 1973 emend. Stover \& Evitt 1978

Senegalinium? dilwynense (Cookson \& Eisenack 1965) Stover \& Evitt 1978 Fig. 6N

Descrição. Cisto proximado circuncavado com compressão dorso-ventral; epicisto maior que o hipocisto; chifre apical curto; chifres antapicais curtos e assimétricos; parede fina, lisa a microgranulada, apresentando dobras verticais; endocisto subpentagonal; paracíngulo indicado por dobras; parassulco indicado por uma leve depressão; arqueopilo intercalar I (2a); opérculo preso; paratabulação indicada pelo arqueopilo e fracamente pelo paracíngulo e parassulco.

Dimensões (4 espécimes). Cisto, a: 59-66 $\mu \mathrm{m}$; l: 53$56 \mu \mathrm{m}$; endocisto, ae: 39-47 $\mu \mathrm{m}$; le: 46-55 $\mu \mathrm{m}$.

\section{Senegalinium obscurum (Drugg 1967) Stover \& Evitt 1978}

Fig. 60

Descrição. Cisto proximado cornucavado com compressão dorso-ventral; chifre apical curto de terminação acuminada; chifres antapicais curtos e assimétricos; parede fina, endocisto oval; paracíngulo indicado por dobras; parassulco indicado por uma leve depressão; arqueopilo intercalar I (2a); opérculo livre; paratabulação indicada pelo arqueopilo e fracamente pelo paracíngulo e parassulco. Dimensões (1 espécime). Cisto, a: $52 \mu \mathrm{m}$; l: $44 \mu \mathrm{m}$; endocisto, ae: $46 \mu \mathrm{m}$; le: $44 \mu \mathrm{m}$.

Gênero Spinidinium Cookson \& Eisenack 1962 emend. Lentin \& Williams 1976; Quattrocchio \& Sarjeant 2003; Sluijs et al. 2009

\section{Spinidinium? clavus Harland 1973}

Fig. 6P-Q

Descrição. Cisto proximado cavado de contorno fusiforme com compressão dorso-ventral; chifre apical curto proeminente; dois chifres antapicais assimétricos, chifre esquerdo maior; perifragma ornamentado por cristas parassuturais, extremidades distais das cristas ornamentadas por espinhos; endocisto subesférico; paracíngulo e parassulco indicados por feições parassuturais; arqueopilo intercalar duvidoso; paratabulação indicada pelas feições parassuturais e arqueopilo.

Dimensões (1 espécime). Cisto, a: $71 \mu \mathrm{m}$; l: $42 \mu \mathrm{m}$; endocisto, ae: $49 \mu \mathrm{m}$; le: $39 \mu \mathrm{m}$; (cristas): 1,5-2 $\mu \mathrm{m}$; $\mathrm{x}$ (espinhos): $1 \mu \mathrm{m}$.

\section{Spinidinium lanterna Cookson \& Eisenack 1970} Fig. 6R

Descrição. Cisto proximado cornucavado de con torno romboidal com compressão dorso-ventral; chifre apical curto; dois chifres antapicais assimétricos, chifre esquerdo maior; epicisto maior que hipocisto pericisto; superfície parassutural; perifragma liso a granulado, contornado por cristas ornamentadas com espinhos, cones e báculas; endocisto subésferico; paracíngulo indicado por cristas ornamentadas; parassulco indicado por uma leve depressão; arqueopilo intercalar I (2a); opérculo preso; paratabulação indicada pelas feições parassuturais e arqueopilo.

Dimensões (1 espécime). Cisto, a: $47 \mu \mathrm{m}$; l: $34 \mu \mathrm{m}$; $\mathrm{x}$ (espinhos): 0,5-1 $\mu \mathrm{m}$.

Gênero Trithyrodinium Drugg 1967 emend. Davey 1969; Lentin \& Williams 1976

\section{Trithyrodinium evittii Drugg 1967} Fig. 7A

Descrição. Cisto proximado cornucavado com compressão dorso-ventral; chifre apical curto de terminação acuminada; chifres antapicais curtos e assimétricos; perifragma fino e delicado; endofragma composto por um endocisto subesférico com parede espessa; parassulco e paracíngulo indistintos; arqueopilo intercalar composto tipo 3I/3I; endopérculo livre; paratabulação indicada pelo endoarqueopilo.

Dimensões (4 espécimes). Endocisto, ae: 79-119 $\mu \mathrm{m}$; le: $69-127 \mu \mathrm{m}$.

Comentário. 0 perifragma não foi observado nos exemplares identificados, possivelmente por problemas de preservação.

Gênero Vozzhennikovia Lentin \& Williams 1976

Vozzhennikovia mariae (Aurisano 1984) Sluijs et al. 2009 


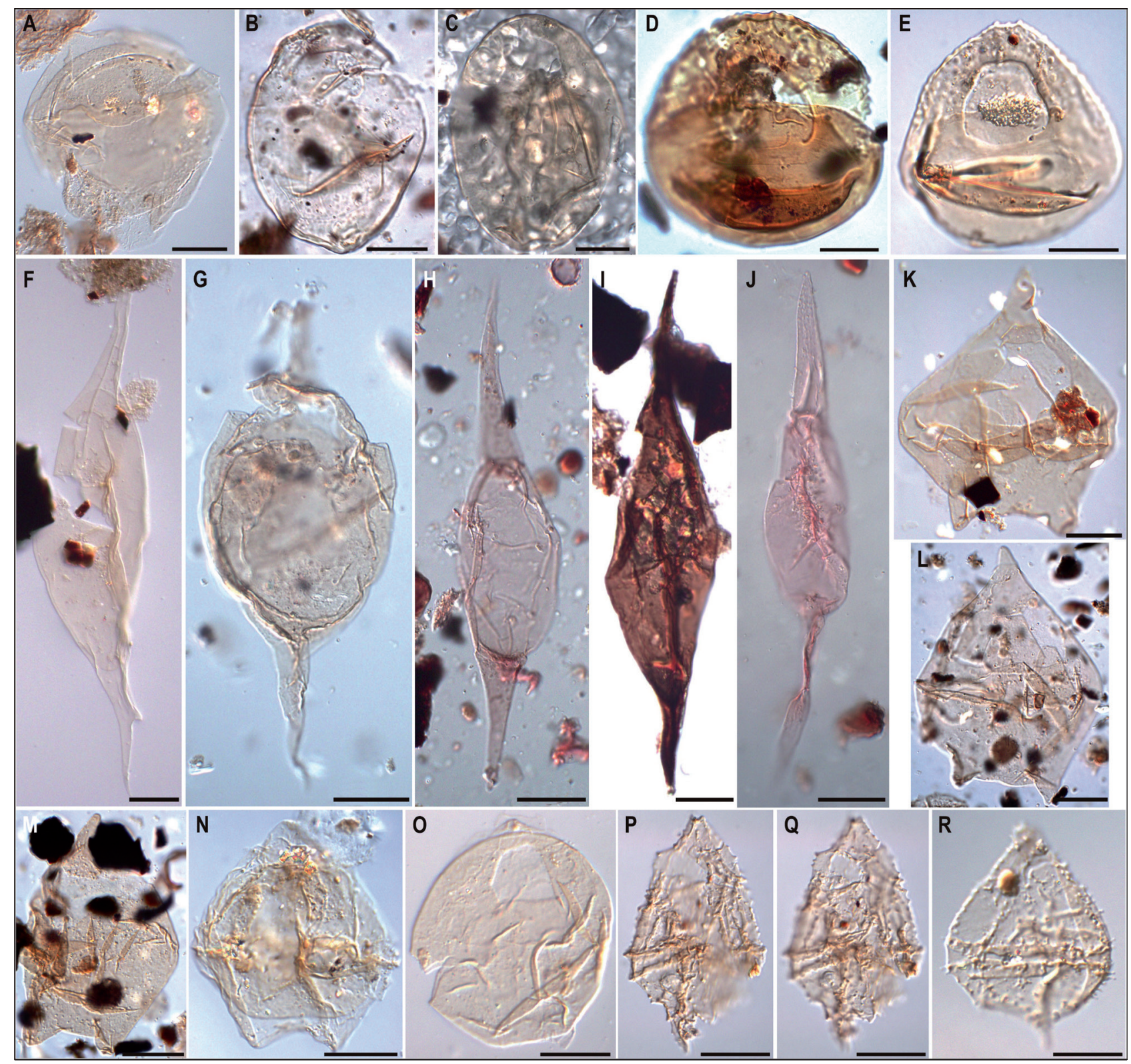

Figura 6. Fotomicrografias de cistos de dinoflagelados peridinioides selecionados (escala gráfica equivalente a $20 \mu \mathrm{m}$ ). A) Manumiella seelandica (poço BP-02, profundidade 2.934,50 m, coordenada England Finder Q40-4); B-C) Manumiella seymourensis (B: BP-02, 3.300-3.315 m, T42, C: BP-02, 3.420-3.435 m, 045); D) Nelsoniella aceras (BP-02, 4.020-4.035 m, E46); E) Nelsoniella tuberculata (BP-02, 4.080-4.095 m, N34-4); F) Palaeocystodinium australinum (BP-02, 2.928,80 m, L45); G) Palaeocystodinium bulliforme (BP-02, 3.150-3.165 m, 048); H) Palaeocystodinium golzowense (BP-01, 1.296 m, N45-2); I) Palaeocystodinium lidiae (BP-02, 2.924,50 m, S43); J) Palaeocystodinium miocaenicum (BP-01, 1.296 m, 035-2); K-L) Pierceites pentagonus (K: BP-01, 3.840-3.855 m, N46, L: BP-01, 3.787-3.796 m, M36-2); M) Pierceites schizocystis (BP-01, 3.787-3.796 m, Q48-4); N) Senegalinium? dilwynense (BP-02, 2.935,00 m, K49); 0) Senegalinium obscurum (BP-02, 3.210-3.225 m, C37); P-Q) Spinidinium? clavus (BP-01, 3.791,90 m, Q46); R) Spinidinium lanterna (BP-01, 3.791,56 m, W45).

Figure 6. Photomicrographs of selected peridinian dinoflagellate cysts (graphic scale equivalents to $20 \mu \mathrm{m}$ ).

Fig. 7B

Descrição. Cisto proximado cornucavado de contorno pentagonal com compressão dorso-ventral; chifre apical curto; dois chifres antapicais assimétricos, chifre esquerdo maior; perifragma ornamentado por espinhos atabulares, extremidades distais dos espinhos acuminadas ou bifurcadas; endocisto subesférico com parede levigada; paracíngulo indicado por finas dobras; parassulco indicado por uma leve depressão contornada por espinhos; arqueopilo intercalar I (2a); opérculo preso; paratabulação indicada pelo arqueopilo e fracamente pelo paracíngulo e parassulco.

Dimensões (2 espécimes). Cisto, a: 58-63 $\mu \mathrm{m}$; 1: 45$49 \mu \mathrm{m}$; endocisto, ae: 39-45 $\mu \mathrm{m}$; le: $41-47 \mu \mathrm{m}$; $\mathrm{x}$ (espinhos): 0,5-2 $\mu \mathrm{m}$.

Vozzhennikovia rotunda (Wilson 1967) Lentin \& Williams 1976

Fig. 7C 
Descrição. Cisto proximado a próximo-condensado cornucavado com compressão dorso-ventral; chifre apical pequeno de terminação truncada; chifre antapical pequeno com terminações cônicas; endocisto subesférico; parede coberta por espinhos; paracíngulo indicado por um alinhamento dos espinhos; parassulco indistinto; arqueopilo intercalar tipo I (2a) iso-deltaforme; opérculo livre; paratabulação indicada pelo paracíngulo e arqueopilo. Dimensões (5 espécimes). Cisto, a: 48-56 $\mu \mathrm{m}$; l: 41-49 $\mu \mathrm{m}$; endocisto, ae: 37-46 $\mu \mathrm{m}$; L: 40-44 $\mu \mathrm{m}$; $\mathrm{x}$ (processos): 1,5-3 $\mu \mathrm{m}$; aca (chifre apical): 4-7 $\mu \mathrm{m}$.

\section{Subfamília Wetzelielloideae (Vozzhennikova 1961) Bujak \& Davies 1983}

Gênero Apectodinium (Costa \& Downie 1976) Lentin \& Williams 1977 emend. Williams et al. 2009

Apectodinium homomorphum (Deflandre \& Cookson 1955) Lentin \& Williams 1977 emend. Harland 1979

Fig. 7D

Descrição. Cisto próximo-condensado cavado com compressão dorso-ventral; chifres geralmente não distintos; endocisto subesférico; parede coberta por processos atabulares com morfologias variadas, com extremidades truncadas, com ramificações pequenas ou acuminadas; paracíngulo e parassulco indistinto; arqueopilo intercalar tipo I (2a); opérculo livre; paratabulação indicada pelo arqueopilo.

Dimensões (6 espécimes). Cisto, a: 66-92 $\mu \mathrm{m}$; l: 60 $98 \mu \mathrm{m}$; endocisto, ae: 47-72 $\mu \mathrm{m}$; le: 47-72 $\mu \mathrm{m} ; \mathrm{x}$ (processos): 3-17 $\mu \mathrm{m}$.

\section{Apectodinium quinquelatum (Williams \& Downie} 1966) Costa \& Downie 1979

Fig. 7E

Descrição. Cisto próximo-condensado cornucavado de contorno pentagonal com compressão dorso-ventral; um chifre apical, dois chifres laterais, dois chifres antapicais assimétricos; perifragama fino coberto por processos atabulares com morfologias variadas, com extremidades truncadas, com ramificações pequenas ou acuminadas; endocisto pentagonal, liso a finamente granulado; paracíngulo e parassulco indistinto; arqueopilo intercalar tipo I (2a); opérculo livre; paratabulação indicada pelo arqueopilo.

Dimensões (1 espécime). Cisto, a: $106 \mu \mathrm{m}$; l: 98 $\mu \mathrm{m}$; endocisto, ae: $65 \mu \mathrm{m}$; le: $71 \mu \mathrm{m}$; x (processos):
4-5 $\mu \mathrm{m}$.

Gênero Dracodinium Gocht 1955 emend. Bujak et al. 1980; Williams et al. 2015

Dracodinium varielongitudum (Williams \& Downie 1966) Costa \& Downie 1979 Fig. 7F

Descrição. Cisto cornucavado a circuncavado com compressão dorso-ventral; um chifre apical, dois chifres laterias bem desenvolvidos, dois chifres antapicais assimétricos, sendo o direito maior; perifragama finamente granulado, com processos simples, distalmente abertos ou fechados; endocisto subesférico, de parede espessa e fortemento granulado; paracíngulo e parassulco indistinto; arqueopilo intercalar tipo I (2a), latiepaliforme; opérculo livre ou preso; paratabulação indicada pelo arqueopilo.

Dimensões (3 espécimes). Cisto, a: 112-125 $\mu \mathrm{m}$;

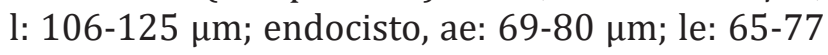
$\mu \mathrm{m} ; \mathrm{x}$ (processos): 4-9 $\mu \mathrm{m}$.

\section{Gênero Petalodinium Williams et al. 2015}

\section{Petalodinium waipawaense (Wilson 1967) Willia- ms et al. 2015 \\ Fig. 7G}

Descrição. Cisto circuncavado de contorno romboidal com compressão dorso-ventral; um chifre apical, dois chifres laterias, dois chifres antapicais, sendo o direito maior; margem do pericisto lisa a serrilhada, com pequenas projeções cônicas perto dos chifres; perifragma finamente granulado; endocisto esférico a subesférico finamente granulado; paracíngulo e parassulco indistinto; arqueopilo intercalar tipo I (2a), latiepeliforme; opérculo preso; paratabulação indicada pelo arqueopilo. Dimensões (4 espécimes). Cisto, a: 118-92 $\mu \mathrm{m}$; l: 96-144 $\mu \mathrm{m}$; endocisto, ae: 61-84 $\mu \mathrm{m}$; le: 66-83 $\mu \mathrm{m}$.

\section{Gênero Piladinium Williams et al. 2015}

\section{Piladinium columnum (Michoux 1988) Williams et al. 2015}

Fig. 7H

Descrição. Cisto cornuvado de contorno romboidal com compressão dorso-ventral; um chifre apical, dois chifres laterias, dois chifres antapicais, sendo o direito maior; perifragma e endofragama recobertos por processos alinhados, ligados distalmente por uma fina e contínua membrana; endocisto 
romboidal e ornamentado por processos; paracíngulo distinto; parassulco indistinto; arqueopilo intercalar tipo I (2a), latiepeliforme; opérculo livre, eventualmente preso; paratabulação indicada pelos processos e arqueopilo.

Dimensões (3 espécimes). Cisto, a: 102-116 $\mu \mathrm{m}$; l: 97-109 $\mu \mathrm{m}$; endocisto, ae: 64-70 $\mu \mathrm{m}$; le: $62-65 \mu \mathrm{m}$.

\section{Piladinium edwardsii (Wilson 1967) Williams et al. 2015}

Fig. 7I

Descrição. Cisto circuncavado de contorno romboidal com compressão dorso-ventral; um chifre apical, dois chifres laterias, dois chifres antapicais, sendo o direito bem desenvolvido, chifre esquerdo pequeno ou indistinto; perifragama recoberto por processos ligados distalmente por uma fina e contínua membrana; endocisto esférico de superficie lisa; paracíngulo e parassulco indistinto; arqueopilo intercalar tipo I (2a), latiepeliforme; opérculo preso; paratabulação indicada pelo arqueopilo. Dimensões (2 espécimes). Cisto, a: 106-124 $\mu \mathrm{m}$; l: $102-119 \mu \mathrm{m}$; endocisto, ae: $68-76 \mu \mathrm{m}$; le: $72-77$ $\mu \mathrm{m}$.

\section{Gênero Rhadinodinium Williams et al. 2015}

Rhadinodinium glabrum (Cookson 1956) Williams et al. 2015

Fig. 7J

Descrição. Cisto proximado circuncavado de contorno romboidal com compressão dorso-ventral; chifres relativamente curtos, um chifre apical, dois chifres laterias, dois chifres antapicais assimétricos, sendo um deles reduzido ou ausente; perifragama liso a finamente granulado; endocisto esférico, escabrado a granulado; paracíngulo fracamente distinto; parassulco indistinto; arqueopilo intercalar tipo I (2a), hiperepeliforme; opérculo livre; paratabulação indicada pelo paracíngulo e arqueopilo.

Dimensões (2 espécimes). Cisto, a: $119-138 \mu \mathrm{m}$; l: 115-130 $\mu \mathrm{m}$; endocisto, ae: 73-102 $\mu \mathrm{m}$; le: 73-96 $\mu \mathrm{m}$.

\section{Gênero Stichodinium Williams et al. 2015}

Stichodinium? lineidentatum (Deflandrea \& Cookson 1955) Williams et al. 2015

Fig. 7L-M

Descrição. Cisto circuncavado de contorno romboidal com compressão dorso-ventral; um chifre apical, dois chifres laterias, dois chifres antapicais, sendo o direito maior; margem do pericisto serrilhada, com pequenas projeções cônicas perto dos chifres; perifragma finamente granulado, ornamentado por tubérculos alinhados refletindo uma paratabulação; endocisto esférico a subesférico finamente granulado; paracíngulo indicado pelo alinhamento da ornamentação; parassulco indistinto; arqueopilo intercalar tipo I (2a), latiepeliforme; opérculo preso; paratabulação indicada pelas feições parassuturais e arqueopilo.

Dimensões (2 espécimes). Cisto, a: 117-120 $\mu \mathrm{m}$; l: $119-126 \mu \mathrm{m}$; endocisto, ae: $80-82 \mu \mathrm{m}$; le: $67-80$ $\mu \mathrm{m}$.

Gênero Wetzeliella Eisenack 1938 emend. Williams \& Downie 1966; Lentin \& Williams 1976;

Williams et al. in Fensome et al. 2009

\section{Wetzeliella articulata Wetzel in Eisenack 1938} Fig. $7 \mathrm{~N}$

Descrição. Cisto circuncavado de contorno pentagonal com compressão dorso-ventral; um chifre apical, dois chifres laterias, dois chifres antapicais assimétricos; superfície atabular composta de inúmeros processos semelhantes a espinhos com terminações truncadas; perifragma liso; endocisto esférico a subesférico, liso a finamente granulado; paracíngulo e parassulco indistintos; arqueopilo intercalar tipo I (2a), soleiforme; opérculo preso; paratabulação indicada pelo arqueopilo.

Dimensões (2 espécimes). Cisto, a: 128-136 $\mu \mathrm{m}$; l: $125-128 \mu \mathrm{m}$; endocisto, ae: $90-91 \mu \mathrm{m}$; le: $85-91$ $\mu \mathrm{m} ; \mathrm{x}$ (espinhos) 3-8 $\mu \mathrm{m}$.

Família Protoperidiniaceae Bujak \& Davies 1983 ex Bujak \& Davies 1983 in Fensome et al. 1998

Subfamília Protoperidinioideae (Autonym)

\section{Gênero Barssidinium Lentin, Fensome \& Williams 1994}

\section{Barssidinium sp. 1}

Fig. 70-Q

Descrição. Cisto próximo-condensado acavado de contorno subesférico a circular com compressão dorso-ventral; parede lisa a finamente granulada; processos cilíndricos atabulares, homogêneos na forma e no tamanho, estriados, extremidades distais pouco desenvolvidas, bordas não ornamentadas; paracíngulo e parassulco indistintos; arqueopilo intercalar tipo I (2a); opérculo preso; paratabulação indicada pelo arqueopilo. 
Dimensões (6 espécimes). Cisto, a: 48-96 $\mu \mathrm{m}$; l: 47$89 \mu \mathrm{m}$; corpo central, ae: 41-76 $\mu \mathrm{m}$; le: $42-72 \mu \mathrm{m}$; (processos): 4-8 $\mu \mathrm{m}$.

Comparações. Barssidinium sp. 1 é similar a Barssidinium evangelineae Lentin et al. 1994 em forma e tamanho, e por apresentar processos estriados; entretanto, não apresenta processos com terminações distais mais desenvolvidadas e ornamentadas.

Comentário. Em muitos exemplares o arqueopilo é de difícil distinção.

Gênero Lejeunecysta Artzner \& Dorhofer 1978 emend. Kjellstrom 1972; Lentin \& Williams 1976; Bujak in Bujak et al. 1980

\section{Lejeunecysta beninensis Biffi \& Grignani 1983} Fig. 7R

Descrição. Cisto proximado acavado de contorno pentagonal com compressão dorso-ventral; epicisto de formato cônico menor que hipocisto; hipocisto alongado de formato trapezoidal; chifres antapicais curtos, depressão antapical moderada; autofragma liso; paracíngulo distinto; parassulco indistinto; arqueopilo tipo I (2a); opérculo livre; paratabulação indicada pelo paracíngulo e arqueopilo.

Dimensões (1 espécime). a: $86 \mu \mathrm{m}$; l: $76 \mu \mathrm{m}$.

Lejeunecysta cinctoria (Bujak in Bujak et al. 1980) Lentin \& Williams 1981

Fig. 7S

Descrição. Cisto proximado acavado de contorno pentagonal com compressão dorso-ventral; epicisto e hipocisto simétricos; chifre apical curto; chifres antapicais curtos e simétricos; autofragma fino, levigado a escabrado; paracíngulo distinto e ornamentado por pequenos espinhos; parassulco indistinto; arqueopilo tipo I (2a); opérculo livre; paratabulação indicada pelo paracíngulo e arqueopilo.

Dimensões (1 espécime). a: $66 \mu \mathrm{m}$; l: $72 \mu \mathrm{m}$; x (espinhos): 0,5-1 $\mu \mathrm{m}$.

\section{Lejeunecysta communis Biffi \& Grignani 1983} Fig. 7T

Descrição. Cisto proximado acavado de contorno pentagonal com compressão dorso-ventral; epicisto e hipocisto simétricos; epicisto cônico, com lados retos; hipocisto trapezoidal; chifre apical curto com extremidade distal sólida; chifres antapicais cônicos com extremidade distal sólida, depressão antapical proeminente; autofragma levigado; paracíngulo distinto; parassulco indistinto; arqueopilo tipo I (2a); opérculo livre; paratabulação indicada pelo paracíngulo e arqueopilo.

Dimensões (1 espécime). a: $57 \mu \mathrm{m}$; l: $60 \mu \mathrm{m}$.

\section{Lejeunecysta globosa Biffi \& Grignani 1983} Fig. 8A-B

Descrição. Cisto proximado acavado de contorno arredondado a pentagonal com compressão dorso-ventral; epicisto e hipocisto relativamente simétricos; epicisto arredontdo, com uma pequena saliência apical; chifres antapicais representados por duas saliências, depressão antapical quase inexistente; autofragma levigado a micropunctado; paracíngulo distinto; parassulco indistinto; arqueopilo tipo I (2a); opérculo livre; paratabulação indicada pelo paracíngulo e arqueopilo.

Dimensões (2 espécimes). a: 43-81 $\mu \mathrm{m}$; l: 51-74 $\mu \mathrm{m}$.

\section{Lejeunecysta hyalina (Gerlach 1961) Artzner \& Dorhofer 1978}

Fig. 8C

Descrição. Cisto proximado acavado de contorno subpentagonal com compressão dorso-ventral; epicisto e hipocisto simétricos de lados arredondados; chifre apical curto; chifres antapicais curtos, depressão antapical proeminente; autofragma fino, liso a finamente granulado, com dobras verticasis irregulares; paracíngulo e parassulco distintos; arqueopilo tipo I (2a); opérculo livre ou preso; paratabulação indicada pelo paracíngulo, parassulco e arqueopilo.

Dimensões (1 espécime). a: $87 \mu \mathrm{m}$; l: $92 \mu \mathrm{m}$.

\section{Lejeunecysta pulchra Biffi \& Grignani 1983} Fig. 8D

Descrição. Cisto proximado acavado de contorno arredondado a pentagonal com compressão dorso-ventral; epicisto e hipocisto relativamente simétricos; chifre apical curto e distalmente acuminado; chifres antapicais curtos e simétricos com extremidas sólidas e acuminadas, depressão antapical proeminente entre os chifres; autofragma levigado; paracíngulo distinto; parassulco indistinto; arqueopilo tipo I (2a); opérculo livre; paratabulação indicada pelo paracíngulo e arqueopilo. Dimensões (1 espécime). a: $67 \mu \mathrm{m}$; l: $63 \mu \mathrm{m}$. 


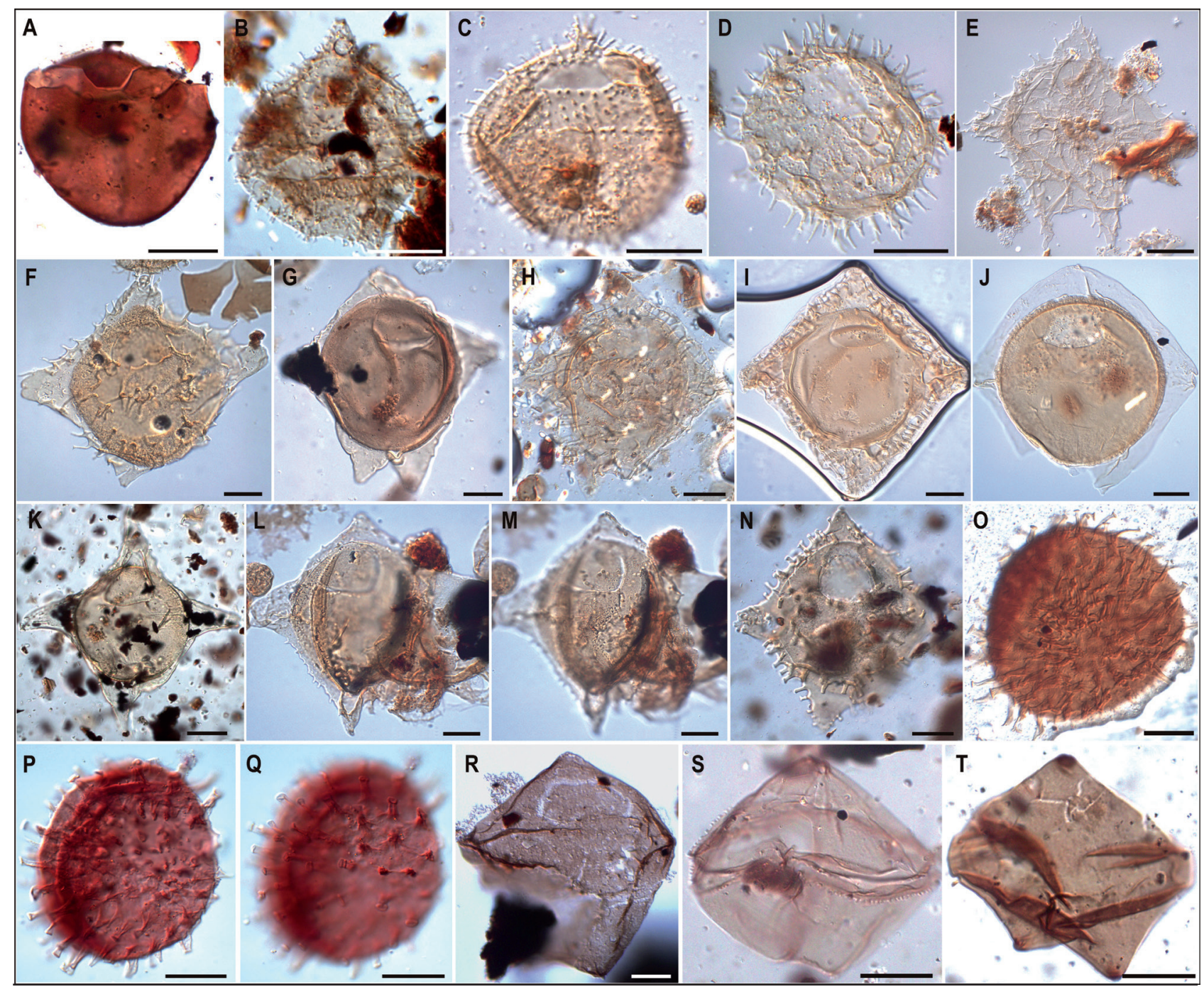

Figura 7. Fotomicrografias de cistos de dinoflagelados peridinioides selecionados (escala gráfica equivalente a $20 \mu \mathrm{m}$ ). A) Trithyrodinium evittii (poço BP-02, profundidade 3.270-3.285 m, coordenada England Finder 034-3); B) Vozzhennikovia mariae (BP-01, 4.080-4.095 m, W38-3); C) Vozzhennikovia rotunda (BP-01, 2.850-2.865 m, R38-2); D) Apectodinium homomorphum (BP-02, 2.745-2.760 m, N34-4); E) Apectodinium quinquelatum (BP-02, 3.000-3.015 m, L39-4); F) Dracodinium varielongitudum (BP-02, 2.715-2.730 m, D43-1); G) Petalodinium waipawaense (BP-02, 2.700-2.715 m, K33-1); H) Piladinium columnum (BP-01, 2.910-2.925 m, N43-3); I) Piladinium edwardsii (BP-01, 3.360-3.375 m, P43); J) Rhadinodinium glabrum (BP-01, 2.571-2.586 m, N42); K) Rhadinodinium sp. (BP-02, 2.640-2.655 m, M29-2); L-M) Stichodinium? lineidentatum (BP-02, 2.775-2.790 m, N41-3); N) Wetzeliella articulata (BP-02, 2.670-2.685 m, F36); 0-Q) Barssidinium sp. 1 (0: BP-01, 936 m, 031-4, P-Q: BP-01, 1.555-1.570 m, P52-1); R) Lejeunecysta beninensis (BP-02, 2.895-2.910 m, S36-4); S) Lejeunecysta cinctoria (BP-02, 1.650-1.665 m, H41-4); T) Lejeunecysta communis (BP-02, 2.130-2.145 m, K45).

Figure 7. Photomicrographs of selected peridinian dinoflagellate cysts (graphic scale equivalents to $20 \mu \mathrm{m}$ ).

Gênero Phelodinium Stover \& Evitt 1978 emend. Mao Shaozhi \& Norris 1988

\section{Phelodinium sp. 1}

Fig. 8E

Descrição. Cisto cornucavado de contorno pentagonal com compressão dorso-ventral; epicisto menor que o hipocisto; chifre apical curto com terminação cônica; chifres antapicais assimétricos, chifre direito maior com terminação cônica; parede fina e lisa; paracíngulo distinto; parassulco indistinto; arqueopilo intercalar tipo I (2a); opérculo livre, ocasionalmente preso; paratabulação indicada pelo arqueopilo e paracíngulo.
Dimensões (2 espécimes). a: 119-133 $\mu$ m; l: 118$119 \mu \mathrm{m}$.

Comparações: Phelodinium sp. 1 é similar a Phelodinium magnificum (Stanley 1965) Stover \& Evitt 1978 em forma e tamanho, entretanto, distingue-se por não apresentam um paracíngulo ornamentado por espinhos.

Gênero Selenopemphix Benedek 1972 emend. Bujak in Bujak et al. 1980, Head 1993

Selenopemphix armata Bujak in Bujak et al. 1980 Fig. 8F

Descrição. Cisto acavado de contorno oval com 
compressão apical-antapical; chifre apical pequeno e arredondado acompanhado de espinhos relativamente longos, chifres antapicais curtos, simétricos e arredondados, acompanhados de espinhos relativamente longos; autofragma levigado a escabrado; parassulco indicado por uma pequena depressão; paracíngulo indicado por cristas onde estão presentes espinhos relativamente longos; arqueopilo intercalar tipo I (2a); opérculo livre ou preso; paratabulação indicada pelo arqueopilo e paracíngulo.

Dimensões (2 espécimes). Cisto, ldv: 93-107 $\mu \mathrm{m}$; l:

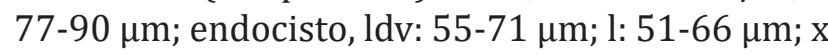
(processos): 11-21 $\mu \mathrm{m}$.

\section{Selenopemphix conspicua (de Verteuil \& Norris} 1992) Louwye, Head \& Schepper 2004

Fig. 8G-H

Descrição. Cisto acavado de contorno suboval a reniforme com compressão apical-antapical; margem parassutural ornamentada; autofragma finamente granulado; paracíngulo ornamentado por processos curtos, extremidades distais truncadas e levemente expandidas; parassulco marcado por uma leve depressão; arqueopilo intercalar tipo I (2a); opérculo livre; paratabulação indicada pelo paracíngulo e arqueopilo.

Dimensões (2 espécimes). Cisto, ldv: 35-60 $\mu \mathrm{m}$; l: 49-67 $\mu \mathrm{m}$; x (processos): 0,5 $\mu \mathrm{m}$.

\section{Selenopemphix brevispinosa Head, Norris \& Mudie} 1989

Fig. 8I-J

Descrição. Cisto acavado de contorno suboval a reniforme com compressão apical-antapical; margem parassutural e pré-cingualr ornamentada; autofragma finamente granulado; paracíngulo ornamentado por processos curtos, extremidades distais expandidas, bifurcadas e denticuladas; parassulco marcado por uma leve depressão; arqueopilo intercalar tipo I (2a); opérculo livre; paratabulação indicada pelo paracíngulo e arqueopilo. Dimensões (2 espécimes). Cisto, ldv: 34-38 $\mu \mathrm{m}$; l: 43-48 $\mu \mathrm{m}$; $\mathrm{x}$ (processos): 1,5-2,5 $\mu \mathrm{m}$.

\section{Selenopemphix dionaeacysta Head, Norris \& Mu- die 1989}

Fig. 8K

Descrição. Cisto acavado de contorno suboval a reniforme com compressão apical-antapical; autofragma liso; ornamentação composta por processos paracíngulares proeminentes, extremidades distais acuminadas, retas ou ramificadas, comumente bifurcadas; parassulco indistinto; arqueopilo intercalar tipo I (2a); opérculo livre, ocasionalmente preso; paratabulação indicada pelo paracíngulo e arqueopilo.

Dimensões (1 espécime). Cisto, ldv: $38 \mu \mathrm{m}$; l: 42 $\mu \mathrm{m}$; $\mathrm{x}$ (processos): 3-4 $\mu \mathrm{m}$.

Selenopemphix nephroides Benedek 1972 emend. Bujak in Bujak et al. 1980; Benedek \& Sarjeant 1981; Head 1993

Fig. 8L

Descrição. Cisto acavado de contorno suboval a reniforme com compressão apical-antapical; chifre apical pequeno e arredondado, chifres antapicais curtos simétricos e arredondados; autofragma levigado a escabrado; parassulco bem marcado por uma depressão; paracíngulo indicado por cristas; arqueopilo intercalar tipo I (2a); opérculo livre; paratabulação indicada pelo paracíngulo e arqueopilo.

Dimensões (4 espécimes). Cisto, ldv: 35-64 $\mu \mathrm{m}$; l: 46-64 $\mu \mathrm{m}$.

Gênero Trinovantedinium Reid 1977 emend. Bujak 1984; de Verteuil \& Norris 1992

\section{Trinovantedinium harpagonium de Verteuil \& Norris 1992}

Fig. 8M-N

Descrição. Cisto acavado próximo-condensado de contorno arredondado a pentagonal com compressão dorso-ventral; chifre apical pouco desenvolvido; assimetria antapical composta por dois lóbulos separados por uma leve depressão; perifragma liso a escabrado; processos predominantemente penitabulares tubiformes a infundibulares; paracíngulo e parassulco distintos; arqueopilo tipo I ( $2 \mathrm{a}$ ) iso-tetaforme a iso-deltaforme; opérculo preso; paratabulação indicada pelos processos penitabulares e arqueopilo.

Dimensões (1 espécime). Cisto, a: $55 \mu \mathrm{m}$; l: $65 \mu \mathrm{m}$; corpo central, a: $54 \mu \mathrm{m}$; l: $47 \mu \mathrm{m}$; x (processos): 4-5 $\mu \mathrm{m}$.

\section{Trinovantedinium variabile (Bujak 1984) de Ver- teuil \& Norris 1992 \\ Fig. 80-P}

Descrição. Cisto acavado próximo-condensado de contorno subeférico com compressão dorso-ventral; chifre apical pouco desenvolvido; assimetria antapical composta por dois lóbulos (nem sempre 
presentes) separados por uma leve depressão; perifragma liso a escabrado; processos morfologicamente variados, extremidades distais acuminadas, abertas ou ramificadas; a disposição dos processos de forma penitabular não é muito clara; paracíngulo e parassulco distintos; arqueopilo tipo I (2a); opérculo preso; paratabulação indicada pelos processos e arqueopilo.

Dimensões (1 espécime). Cisto, a: $76 \mu \mathrm{m}$; l: $66 \mu \mathrm{m}$; corpo central, ae: $56 \mu \mathrm{m}$; le: $51 \mu \mathrm{m}$; (processos): 4-6 $\mu \mathrm{m}$.

\section{Família incertae sedis}

\section{Gênero Octodinium Wrenn \& Hart 1988}

Octodinium askiniae Wrenn \& Hart 1988
Fig. 8R-S

Descrição. Cisto cornucavado de contorno subelíptico a subtriangular; chifre apical longo e fino; chifres antapicais longos e simétricos; perifragma liso, granular ou rugulado; endocisto alongado de contorno subtriangular; paracíngulo fracamente distinto por duas finas dobras; parassulco fracamente distinto por uma leve depressão; arqueopilo intercalar tipo I (2a); paratabulação indicada pelo arqueopilo.

Dimensões (3 espécimes). a: 82-95 $\mu \mathrm{m}$; l: 32-33 $\mu \mathrm{m}$; endocisto, ae: 55-58 $\mu \mathrm{m}$; le: $32-33 \mu \mathrm{m}$.

Comentário. Em todos exemplares identificados os chifres (apical e antapicais) encontraram-se quebrados, impossibilitando medições mais precisas.

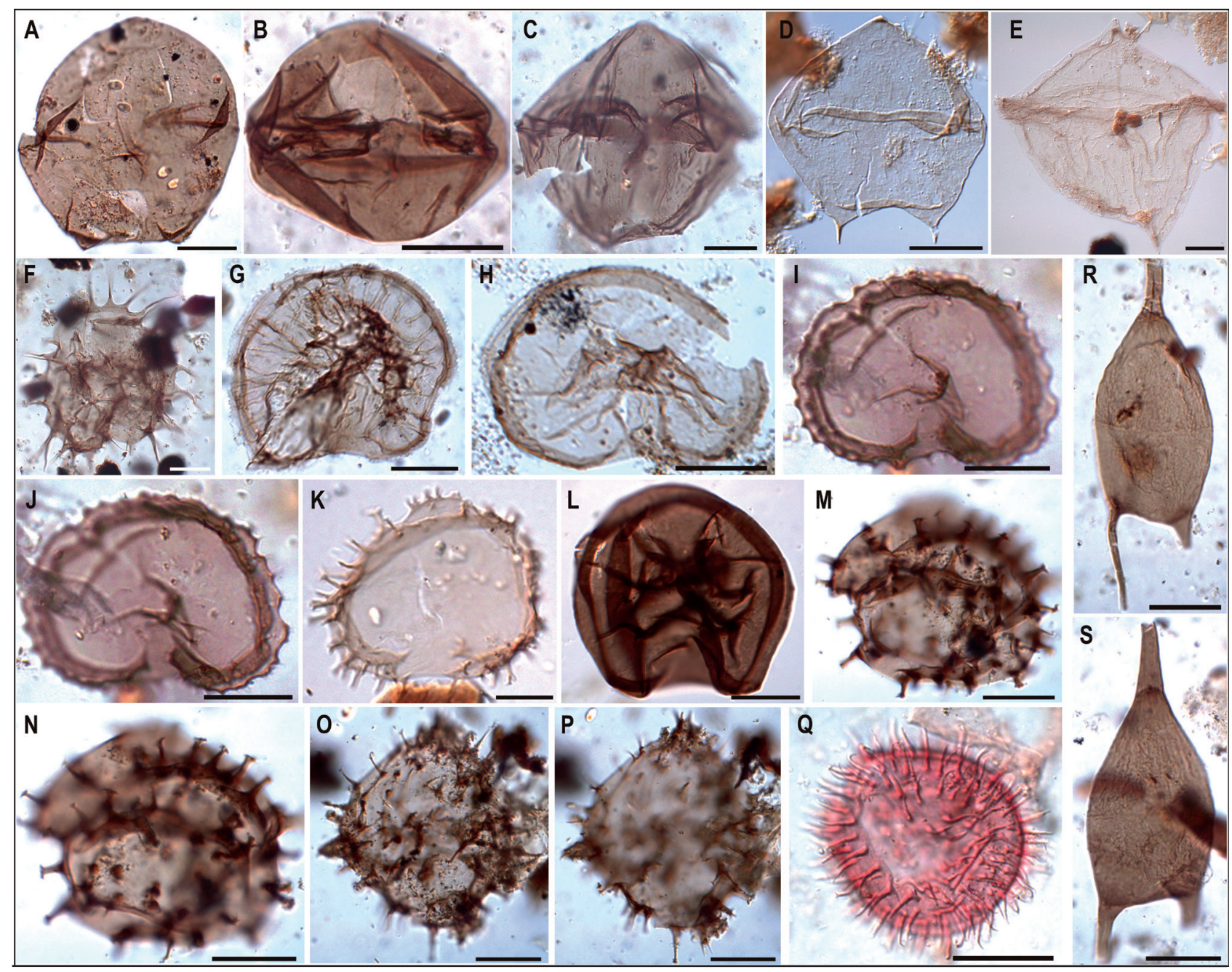

Figura 8. Fotomicrografias de cistos de dinoflagelados peridinioides selecionados (escala gráfica equivalente a $20 \mu \mathrm{m}$ ). A-B) Lejeunecysta globosa (A: poço BP-02, profundidade 1.440-1.455 m, coordenada England Finder G40-3, B: BP-02, 2.070-2.085 m, J47); C) Lejeunecysta hyalina (BP-02, 2.250-2.265 m, S32-2); D) Lejeunecysta pulchra (BP-01, 2.539,43 m, P46); E) Phelodinium sp. 1 (BP-02, 3.060-3.075 m, G36-1); F) Selenopemphix armata (BP-02, 1.500-1.515 m, H45); G-H) Selenopemphix conspicua (G: BP-02, 2.250-2.265 m, K45-2, H: BP-02, 2.265-2.280 m, U44-3); I-J) Selenopemphix brevispinosa (BP-02, 1.230-1.245 m, G521); K) Selenopemphix dionaeacysta (BP-02, 1.170-1.185 m, P44); L) Selenopemphix nephroides (BP-02, 1.680-1.695 m, U44-3); M-N) Trinovantedinium harpagonium (BP-02, 1.440-1.455 m, R45-1); O-P) Trinovantedinium variabile (BP-02, 1.440-1.455 m, M43-3); Q) Echinidinium sp. (BP-01, 1.950 m, Q40-1); R-S) Octodinium askiniae (R: BP-02, 1.860-1.875 m, D37, S: BP-02, 1.860$1.875 \mathrm{~m}, \mathrm{~V} 36-3)$.

Figure 8. Photomicrographs of selected peridinian dinoflagellate cysts (graphic scale equivalents to $20 \mu \mathrm{m}$ ). 


\section{Considerações finais}

Avaliando a composição das associações de cistos de dinoflagelados reconhecidas para o Cretáceo Superior na margem atlântica sul-americana e regiões próximas ao Círculo Polar Antártico, verificou-se que a Bacia de Pelotas comportou associações contendo formas similares àquelas registradas para a margem continental brasileira (e.g. bacias de Santos, Campos e Ceará), como pode ser constatado pelas ocorrências de Nelsoniella aceras, Isabelidinium korojonense e Isabelidinium pellucidum, indentificados anteriormente por Helby et al. (1987), Lana \& Botelho Neto (1989), Arai et al. (2000) e Arai (2007).

Com a exceção de Deflandrea foveolata, que havia sido observada somente nos depósitos da Nova Zelândia (Wilson, 1988; Crouch et al., 2014), elementos de natureza mais cosmopolita típicos de associações paleocênicas foram identificados, incluindo Palaeoperidinium pyrophorum, Pierceites pentagonus, Senegalinium? dilwynense, Isabelidinium? viborgense, Cerodinium striatum, Trithyrodinium evittii e Palaeocystodinium bulliforme (Powell, 1992; Ferreira, 2004; Williams et al., 2004).

Cistos de dinoflagelados atribuídos à Subfamília Wetzelielloideae apresentaram-se diversificados, sendo Apectodinium homomorphum registrado mais comumente. Ademais, foram observadas frequências significativas de espécies pertencentes ao gênero Deflandrea, incluindo táxons de caráter mais endêmico, tais como $D$. antarctica e $D$. convexa, além de Octodinium askiniae, registrados nas altas latitudes do Hemisfério Sul no Eoceno (Williams et al., 2004; Bijl et al., 2011).

Relativamente mais raros nas seções neogenas da bacia, cistos peridinioides foram representados pelas espécies Trinovantedinium harpagonicum, Lejeunecysta globosa, Selenopemphix dionaeacysta e Selenopemphix nephroides, cujo registro estratigráfico tem sido observado em diversas localidades do Oceano Atlântico entre o Mioceno e o Plioceno (de Verteuil \& Norris, 1992; de Verteuil, 1996; Williams et al., 2004; Silva et al., 2011).

De forma inédita, este trabalho compreende o detalhamento taxonômico dos cistos de dinoflagelados peridiniodes registrados em intervalos estratigráficos ainda não analisados da bacia. Entre os resultados mais expressivos, ressalta-se o considerável número de espécies documentadas, algumas das quais, amplamente empregadas nos biozoneamentos mundiais. Estas informações estão sendo utilizadas para elaboração de um arcabouço palinoestratigráfico próprio para a Bacia de
Pelotas, o qual constituirá tema de contribuição subsequente dos autores deste trabalho.

Agradecimentos. 0 primeiro autor agradece ao Programa de Fomento à Pesquisa (PROPESQUFRGS). Este trabalho faz parte da tese de doutorado do primeiro autor, junto ao Programa de Pós-Graduação em Geociências da Universidade Federal do Rio Grande do Sul.

\section{Referências}

Anjos, G.S. \& Carreño, A.L. 2004. Bioestratigrafia (Foraminiferida) da sondagem 1-SCS-3B, Plataforma de Florianópolis, Bacia de Pelotas. Revista Brasileira de Paleontologia, 7(2): 127-138.

Anjos-Zerfass, G.S., Souza, P.A. \& Chemale Jr., F. 2008. Biocronoestratigrafia da Bacia de Pelotas: estado atual e aplicação na geologia do petróleo. Revista Brasileira de Geociências, 38(2): 47-62.

Arai, M. 2005. Histórico da Palinoestratigrafia marinha no Brasil com ênfase em dinoflagelados cretáceos. Arquivos do Museu Nacional, 63: 371-384.

Arai, M. 2007. Sucessão das associações de dinoflagelados (Protista, Pyrrhophyta) ao longo das colunas estratigráficas do Cretáceo das bacias da margem continental brasileira: uma análise sob ponto de vista paleoceanográfico e paleobiogeográfico. Porto Alegre, 241p. Tese de Doutorado, Programa de Pós-graduação em Geociências, Instituto de Geociências, Universidade Federal do Rio Grande do Sul.

Arai, M. 2014. Aptian/Albian (Early Cretaceous) paleogeography of the South Atlantic: a paleontological perspective. Brazilian Journal of Geology, 44: 339350.

Arai, M., Botelho Neto, J., Lana, C.C. \& Pedrão, E. 2000. Cretaceous dinoflagellate provincialism in Brazilian marginal basins. Cretaceous Research, 21: 351-366.

Arai, M., Masure, E. \& Lemos, V.B. 2006. Occurrence of a high-diversity Aptian microphytoplanktonic assemblage in Pelotas Basin (Southern Brazil): its implication for the Early Cretaceous history of the South Atlantic. In: SIMPÓSIO DO CRETÁCEO DO BRASIL, 7. e SIMPÓSIO DO TERCIÁRIO DO BRASIL, 1., 2006, Serra Negra. Boletim de resumos... Rio Claro, p. 12.

Askin, R.A. 1988. Campanian to Paleocene palynological succession of Seymour and adjacent islands, north-eastern Antarctic Peninsula. Geological Society of America Memoir, 169: 131-153.

Bijl, P.K., Pross, J., Warnaar, J., Stickley C.E., Huber, M., Guerstein, R., Houben, A.J.P., Sluijs, A., Visscher, H. \& Brinkhuis, H. 2011. Environmental forcings of Paleogene Southern Ocean dinoflagellate biogeography. Paleoceanography, 26: 1-12.

Bijl, P.K., Sluijs, A. \& Brinkhuis, H. 2013. A magneto- and chemostratigraphically calibrated dinoflagellate cyst zonation of the early Palaeogene South Pacific Ocean. Earth-Science Reviews, 124: 1-13.

Botelho Neto, J. 1996. Porção Norte da plataforma de Regência, Bacia do Espírito Santo: caracterização pa- 
linoestratigráfica e evolução paleoambiental. Rio de Janeiro, 163p. Dissertação de Mestrado, Programa de Pós-graduação em Geologia, Instituto de Geociências, Universidade Federal do Rio de Janeiro.

Buchmann, F.S.C. 1994. Distribuição dos fósseis pleistocênicos na zona costeira e plataforma continental interna no Rio Grande do Sul. Acta Geológica Leopoldensia, 17(39/1): 355-364.

Bueno, G.V., Zacharias, A.A., Oeiro, S.G., Cupertino, J.A., Falkenhein, F.U.H. \& Martins Neto, M.A. 2007. Bacia de Pelotas. Boletim de Geociências da Petrobras, 15(2): 551-559.

Ceolin, D., Fauth, G. \& Coimbra, J.C. 2010. Cretaceous Lower Paleogene Ostracods from the Pelotas Basin, Brazil. Palaeobiodiversity and Palaeoenvironments, 91(2): 111-128.

Closs, D. 1967. Miocene planktonic foraminifera fromsouthern Brazil. Micropaleontology, 13(3): 337-344.

Coimbra, J.C., Carreño, A.L. \& Anjos, G.S. 2009. Biostratigraphy and paleoceanographical significance of the Neogene planktonic foraminifera from the Pelotas Basin, southernmost Brazil. Revue de micropaléontologie, 52: 1-14.

Crouch, E.M., Willumsen, P.S., Kulhanek, D. \& Gibbs, S. 2014. A revised Paleocene (Teurian) dinoflagellate cyst zonation for eastern New Zealand. Review of Palaeobotany and Palynology, 202: 47-79.

de Verteuil, L. 1996. Data report: Upper Cenozoic dinoflagellate cysts from the continental slope and rise off New Jersey. Proceedings of the Ocean Drilling Program, Scientific Results, 150: 439-454.

de Verteul, L. \& Norris, G. 1992. Miocene Protoperidiniacean dinoflagellate cyst from the Meryland and Virginia coastal plain. In: Head, M.J. \& Wrenn, J.H. (Eds.). Neogene and Quaternary Dinoflagellate Cyst and Acritarchs. Dallas, American Association of Stratigraphic Palynologists, p. 391-430.

Dias J.L., Silveira D.P., Sad, A.R.E. \& Latgé, M.A.L. 1994. Bacia de Pelotas: Estágio atual do conhecimento geológico. Boletim de Geociências da Petrobras, 8(1): 235-245.

Evitt, W.R. 1985. Sporopollenin Dinoflagellate cysts: Their Morphology and Interpretation. Dallas, American Association of Stratigraphic Palynologists Foundation, 333p.

Fensome, R.A., Taylor, F.R.J., Norris, G., Sarjeant, W.A.S., Wharton, D.I. \& Williams, G.L. 1993. A Classification of fossil and living dinoflagellates. Micropaleontology Press Special Paper, 7: 1-351.

Fensome, R.A., MacRae, R.A, Moldowan, J.M., Taylor F.J.R. \& Williams, G.L. 1996. The early Mesozoic radiation of dinoflagellates. Paleobiology, 22(3): 329-338.

Ferreira, E.P. 2004. Palinoestratigrafia e caracterização de paleoambientes da seção paleocênica-eocênica da bacia de Sergipe. Rio de Janeiro, 253p. Tese de Doutorado, Programa de Pós-Graduação em Geologia, Instituto de Geociências, Universidade Federal do Rio de Janeiro.

Fischer, T.V, Souza, P.A., Helenes, J. \& Arai, M. 2013. Associações palinológicas do Paleogeno da Bacia de Pelotas (Poço BP-1, Brasil) e seu significado estrati- gráfico. Geociências, 32 (4): 677-695.

Fontana, R.L. 1990. Investigações geofísicas preliminares sobre o Cone do Rio Grande, Bacia de Pelotas, Brasil. Acta Geológica Leopoldensia, 13(6): 161-170.

Fontana R.L. 1996. Geotectônica e sismoestratigrafia da Bacia de Pelotas e Plataforma de Florianópolis. Porto Alegre, 214p. Tese de Doutorado, Programa de Pós-graduação em Geociências, Instituto de Geociências, Universidade Federal do Rio Grande do Sul.

Gomide, J. 1989. Bacia de Pelotas - Biocronoestratigrafia baseada em nanofósseis calcáreos. In: CONGRESSO BRASILEIRO DE PALEONTOLOGIA, 11., 1989. Curitiba. Anais...Curitiba, SBG, p. 338-351.

Gonçalves, A., Oliveira, M.A.M. \& Motta, S.0. 1979. Geologia da Bacia de Pelotas e da Plataforma de Florianópolis. Boletim Técnico da Petrobrás, 22(3): 157-174.

Gradstein, F.M., Ogg, J.G., Schmitz, M.D. \& Ogg, G.M. 2012. The Geologic Time Scale. Amsterdam, Elsevier, $1.172 \mathrm{p}$.

Guerra, R.M., Tokutake, L.R. \& Fauth, G. 2010. Upper Campanian calcareous nannofossils from a core of well 2-RSS-1, Pelotas Basin, Brazil. Revista Brasileira de Paleontologia, 13(3): 181-188.

Guerra, R.M., Tokutake, L.R. \& Fauth, G. 2012. Cretaceous calcareous nannofossils from Pelotas Basin, Brazil: biostratigraphic and paleoecological inferences. Journal of South American Earth Sciences, 36: 55-71.

Guerstein, G.R. \& Junciel, G.L. 2001. Quistes de dinoflagelados del Cenozoico de la Cuenca del Colorado, Argentina. Ameghiniana, 38: 299-316.

Guerstein, G.R., Guler, M.V., Williams, G.L., Fensome, R.A. \& Chiesa, J.O. 2008. Mid Palaeogene dinoflagellate cysts from Tierra del Fuego, Argentina: biostratigraphy and palaeoenvironments. Journal of Micropalaeontology, 27: 75-94.

Guler, M.V. \& Guerstein, G.R. 2003. Quistes de dinoflagelados Cladopyxiaceae, Gonyaulacaceae, Goniodomaceae e incierta del Oligoceno-Plioceno temprano de la cuenca del Colorado, Argentina. Revista Española de Paleontología, 18(1): 23-47.

Helby, R., Morgan, R. \& Partridge, A.D. 1987. A palynological zonation of the Australian Mesozoic. In: Jell, P.A. (Ed.). Studies in Australian Mesozoic Palynology. Sydney, Association of Australasian Palaeontologists, p. 1-97.

Hermany, G., Souza, P.A. \& Torgan, L.C. 2013. Paleoecologia do sistema Pinguela-Palmital-Malvas, Holoceno da Bacia de Pelotas, RS, Brasil: uma abordagem focada na utilização de análises multivariadas para obtenção de diatomáceas descritoras. Pesquisas em Geociências, 40(1): 31-49.

Jones, R.W. 2011. Applications of Palaeontology: Techniques and Case Studies. New York, Cambridge University Press, 420p.

Koutsoukos, E.A.M. 1982. Geohistória e paleoecologia das bacias marginais de Florianópolis e Santos. In: CONGRESSO BRASILEIRO DE GEOLOGIA, 32., 1982, Salvador. Anais... Salvador, SBG, v.5, p. 2369-2382.

Lana, C.C. 1997. Palinologia e estratigrafia integrada da seção Cenomaniano médio- Turoniano inferior da porção centro-leste da Bacia Potiguar, NE do Brasil. 
Porto Alegre, 144p. Dissertação de Mestrado, Programa de Pós-graduação de em Geociências, Instituto de Geociências, Universidade Federal do Rio Grande do Sul.

Lana, C.C. \& Botelho Neto, J. 1989. Evidências de provincialismo entre os dinoflagelados peridinióides do Cretáceo Superior - Paleoceno das bacias de Santos e Potiguar, Brasil. CONGRESSO BRASILEIRO DE PALEONTOLOGIA, 11., 1989, Curitiba. Anais...Curitiba, SBP, v.1, p. 353-371.

Lana, C.C. \& Pedrão, E.P. 2000. Um episódio de incursão marinha no Eoaptiano (Eoalagoas) da Bacia de Almada, BA, Brasil. Revista Universidade de Guarulhos, Geociências V, 89-92.

Lentin, J.K., Fensome, R.A. \& Williams, G.L. 1994. The stratigraphic importance of species of Sumatradinium, Barssidinium and Erymnodinium, Neogene dinoflagellate genera from offshore eastern Canada. Canadian Journal of Earth Sciences, 31: 567-582.

Milani, E.J., Rangel, H.D., Bueno, G.V., Stica, J.M., Winter, W.R., Caixeta, J.M. \& Neto, O.C.P. 2007. Bacias Sedimentares Brasileiras - Cartas estratigráficas. Boletim de Geociências da Petrobras, 15(2): 183-205.

Powell, A.J. 1992. A Stratigraphic Index of Dinoflagellate Cysts. London, Chapman \& Hall, 290p.

Premaor, E. 2016. Bioestratigrafia das associações de cistos de dinoflagelados do Cretáceo Inferior (Albiano) ao Neogeno (Plioceno) da Bacia de Pelotas, Brasil. Porto Alegre, 331p. Tese de Doutorado, Programa de Pós-graduação em Geociências, Instituto de Geociências, Universidade Federal do Rio Grande do Sul.

Premaor, E., Souza, P.A., Arai, M. \& Helenes, J. 2010. Palinomorfos do Campaniano (Cretáceo Superior) da Bacia de Pelotas, Rio Grande do Sul: implicações bioestratigráficas e paleoambientais. Pesquisas em Geociências, 37(1): 63-79.

Regali, M.S.P., Uesugui, N. \& Santos, A.S. 1974a. Palinologia dos sedimentos meso-cenozóicos do Brasil. I. Boletim Técnico da Petrobras, 17(3): 177-190.

Regali, M.S.P., Uesugui, N. \& Santos, A.S. 1974b. Palinologia dos sedimentos meso-cenozóicos do Brasil. II. Boletim Técnico da Petrobras, 17(4): 263-301.

Roncaglia, L., Field, B.D., Raine, J.I., Schiøler, P. \& Wilson, G.J. 1999. Dinoflagellate biostratigraphy of Piripauan-Haumurian (Upper Cretaceous) sections from the northeast South Island, New Zealand. Cretaceous Research, 20: 271-314.

Sarkis, M.F. 2002. Caracterização palinoestratigráfica e paleoecológica do limite Cretáceo-Terciário na seção Poty, Bacia de Pernambuco/Paraíba, nordeste do Brasil. Rio de Janeiro, 253p. Tese de Doutorado, Programa de Pós-Graduação em Geologia, Instituto de Geociências, Universidade Federal do Rio de Janeiro.

Silva, W.G, Souza, P.A., Helenes, J. \& Arai, M. 2011. Palinomorfos Neogenos do Poço 2-CA-1-RS, Bacia de Pe- lotas, Brasil: Significado Bioestratigráfico e Paleoecológico. Geologia USP, Série Científica, 11: 149-169.

Silva, W.G., Zerfass, G.S.A., Souza, P.A. \& Helenes, J. 2015. Biochronostratigraphy and paleoenvironment analysis of Neogene deposits from the Pelotas Basin (well 2-TG-96-RS), Southernmost Brazil. Anais da Academia Brasileira de Ciências, 87(3): 1565-1582.

Simões, M.G., Silva, S.A.M., Rodrigues, S.C. \& Coimbra, J.C. 2008. Braquiópodes (Rhynchonelliformea, Bouchardioidea) neogenos da Bacia de Pelotas (RS) e seu significado paleoambiental. Revista Brasileira de Geociências, 38(4): 676-685.

Stanley, E.A. 1965. Upper Cretaceous and Paleocene plant microfossils and Paleocene dinoflagellates and hystrichosphaerids from northwestern South Dakota. Bulletin of American Paleontology, 49(222): 179384.

Stover, L.E. \& Evitt, W.R. 1978. Analyses of pre-Pleistocene organic-walled dinoflagellates. Stanford University Publications, Geological Sciences, 15: 1-300.

Stover, L.E., Brinkhuis, H., Damassa, S.P., de Verteuil, L., Helby, R.J., Monteil, E., Partridge, A.D., Powell, A.J., Riding, J.B., Smelror, M. \& Williams, G.L. 1996. Mesozoic-Tertiary dinoflagellates, acritarchs and prasinophytes. In: Jansonius, J. \& McGregor, D.C. (Eds.). Palynology: Principles and Applications. Dallas, American Association of Stratigraphic Palynologists, p. 641-750.

Thiesen, Z.V. 1977. Bolivinitidae e Caucasinidae (Foraminiferida) do Cenozóico Superior da Bacia de Pelotas, Rio Grande do Sul. Acta Geológica Leopoldensia, 2(3): 3-82.

Uesugui, N. 1979. Palinologia: técnicas de tratamento de amostras. Boletim Técnico da Petrobras, 22(4): 229-240.

Villwock, J.A. \& Tomazelli, L.J. 1995. Geologia Costeira do Rio Grande do Sul. Notas Técnicas, 8: 1-45.

Williams, G.L., Brinkhuis, H., Pearce, M.A., Fensome, R.A. \& Weegink, J.W. 2004. Southern ocean and global dinoflagellate cyst events compared: Index events for the Late Cretaceous-Neogene. Proceedings of the Ocean Drilling Program, Scientific Results, 189: 1-98.

Williams, G.L., Fensome, R.A. \& MacRae, R.A. 2017. The Lentin and Williams index of fossil dinoflagellates 2017 edition. American Association of Stratigraphic Palynologists, Contibutions Series 48, 1097p.

Wilson, G.J. 1988. Paleocene and Eocene dinoflagellate cysts from Waipawa, Hawkes Bay, New Zealand. New Zealand Geological Survey Paleontological Bulletin, 57: 1-96.

Wrenn, J.H. \& Hart, G.F. 1988. Paleogene dinoflagellate cyst biostratigraphy of Seymour Island, Antarctica. Geological Society of America Memoirs, 169: 321447.

Manuscrito 646

Editores: Marina B. Soares \& Maria do Carmo Lima e Cunha 